
3 Research Square
Preprints are preliminary reports that have not undergone peer review.
They should not be considered conclusive, used to inform clinical practice,
or referenced by the media as validated information.

\title{
Climate Change as a Precursor to the Food Security; Empirical Evidence Over Punjab Regions, Pakistan
}

Sohail Abbas

Konkuk University - Seoul Campus: Konkuk University

Shazia Kousar ( $\square$ shaziakousar029@gmail.com)

Lahore College for Women University

Mahr Sahibzad Khan

PMD: Pakistan Meteorological Department

\section{Research Article}

Keywords: Climate change, Wheat yield, Food security, rainfall, ARDL, Punjab regions

Posted Date: October 21st, 2021

DOI: https://doi.org/10.21203/rs.3.rs-852972/v1

License: () (i) This work is licensed under a Creative Commons Attribution 4.0 International License. Read Full License 


\section{Abstract}

Climate change is bringing drastic changes to the food availability, accesses ability, quality, and stability of the food system in the World. This study investigates the impacts of climate change as a precursor on food security over Punjab regions, Pakistan, from 1979-2020. The bound test cointegration approach is applied with auto-regressive distributed lag (ARDL) to investigate the linear impact of climate variables on food production. This study found that Wheat cultivated area, total irrigated area, total un-irrigated area, and total area sown has a positive and significant impact on food production in all regions of Punjab Pakistan. However, while average, the maximum and minimum temperature is negatively and significantly associated with food production in all regions of Punjab except northern Punjab, where maximum temperature affects positively food production Punjab. Rainfall positively affects food production in central and western Punjab while negatively affecting food production in northern and southern Punjab. Furthermore, wind speed negatively affects food production in all regions of Punjab except northern Punjab.

\section{Introduction}

The Agricultural sector is considered the "mainstay of rural Pakistan" and plays a very important role in the economic development of Pakistan. With changing weather conditions and increasing population pressure, the agriculture sector is on the threat (Asian Development Bank, 2010). Adnan et al., (2017) determined that due to changing trends of the vulnerability of climate change, the agriculture sector is at high risk because of its geographical location with arid and semi-arid conditions. Climate change is considered to global phenomenon, which brings variation in rainfall patterns, temperature intensity, and negatively influences water resource management over the world (Ray et al. 2015). Vulnerability in climate exerts pressure on agricultural practices in Pakistan (Ullah, 2017; Nawaz et al., 2019).

The agriculture sector has been affected by variations in temperature and rainfall cycles. Such fluctuations like increasing temperatures in arable areas, changes in rainfall patterns, increased variability of rainfall in the summer monsoon season, and severe water-stressed conditions (such as floods and droughts) have negative impacts on agriculture practices in arid and semi-arid areas cause a decrease in the yield about 6-18\% (Ullah, 2017). Hanif et al. (2010) identified the effects of the climatic change due to variation in hydrological cycles. The negative impacts of the extreme climate were examined on the agriculture sector in Pakistan. IPCC (2007) confirmed the increasing trends of the temperature in the south Asian regions in the summer monsoon season. Such high observed trends of temperature negatively influence agricultural production. The variation in the monsoon rainfall from year to year has a strong and significant impact on the variability of the Wheat crop all over the world (Webster et al., 1998). The variation in the rainfall during the summer season from year to year has a strong and significant impact on the variability of agricultural crops in India (Asada and Matusmoto, 2009).

Wheat is one of the major key staple foods for more than half of the World's population (International Rice Research Institute, 2006; Anonymous, 2013) and supports the livelihoods and economies of several billion people. In Pakistan, Punjab province is considered the major agricultural hub. Wheat and rice contribute to the major sharing in crops of Punjab province. Rice is the second big crop with 70.2 percent after Wheat with 75 percent (Afzal et al., 2017) Several studies have been conducted on the impacts of climate change on wheat crops in Pakistan and India regions (Iqbal et al., 2005; Hussain and Mudasser, 2007; Abbas et al., 2007; Sultana et al., 2009; Janju et al., 2010; Ashfaq et al., 2011; Siddiqui et al., 2012; Tariq et al., 2014; Zulfiqar and Hussain, 2014; Ray et al., 2015; Ahmed et al., 2016; Abdullah et al., 2016; Arshad et al., 2017; Ali et al., 2017; Bokhari et al., 2017; Kirby et al., 2017; Wang et al., 2017; Abid et al., 2018; Ahmad et al., 2019; Gul et al., 2020; Dhamija et al., 2020).

Iqbal et al (2005) identified the ARIMA model for the prediction of the wheat crop area and production in territory of Pakistan between 2002-2022. Abbas et al. (2007) analyzed the food security by the declining trends of the major food wheat crop for the period of 2000-2004. Sultana et al. (2009) measured the vulnerability of climate change and its impacts on wheat production in various climatic zones of Pakistan under various climate change scenarios in the country. Ashfaq et al. (2011) evaluated the impacts of climate variability on wheat productivity in mixed areas of Punjab regions, Pakistan from 1980-81 to 2008-09. Tariq et al. (2014) investigated the effects of climate change on major food wheat crop along with its per capita availability over Pakistan in 19802012. Zulfiqar and Hussain (2014) indicated the gaps of Forecasting wheat production to assess the future food security issues at various levels of consumption in Pakistan through 2013-2025. Abduallah et al., (2016) examined the impacts of warming climatic conditions on major food crop availability on the Pakistan from 1984-2014.

The negative impacts on the Climate were noticed on humans regarding the food security issues. Ahmed et al. (2016) studied the Calibration and validation of APSIM-Wheat and CERES-Wheat for spring wheat under various rainfed conditions over Pakistan. Abid et al. (2018) determined the trends and variation in wheat crop over country level during 1981-85 to 2011-15. Ahmad et al. (2019) exhibits the impact of Climatic change on various stages of the of rice-wheat cropping system in Punjab, Pakistan through 1980-2014. Gul et al. (2020) explored the influence of climate change on yield of various wheat cultivars under various agro-environmental circumstances using the crop growth model in Khyber Pakhtunkhwa, Pakistan. There is no empirical study was reported on the impacts of the Climate change on the major food crop wheat production and food security on the various regions of Pakistan.

Agriculture land is approximately 60 million acres, of which $51 \%$ is cultivated, and $9.5 \%$ is laying as cultivated unused land in different parts of Punjab. Rice has contributed the $2.7 \%$ in the value added to agriculture in Pakistan, which is the second source of food in Punjab (Government of Pakistan, 2013; Abbas and Dastgeer, 2021b). The agriculture sector of Punjab has already been under stress due to changing climate, risk of desertification, and rising demand for food. Therefore, additional empirical based study is needed to measure the effects of climate change on wheat crop in the various region of Punjab, Pakistan (Abbas et al., 2021a). The importance of the agriculture sector in the Pakistan economy, the effect of rainfall and temperature on the wheat yield region-wise is important to investigate. The main objective of this study is to determine the impacts of the climate change and on the major food crop wheat production and food security on the various regions of Pakistan.

\section{Data And Methodology}




\section{Study area- Regional features}

The Punjab province lies between latitudes $31.17^{\circ} \mathrm{N}$ and longitudes $72.70^{\circ} \mathrm{E}$. Punjab is the 2nd biggest province of Pakistan after Baluchistan, with an area of $205,344 \mathrm{~km}^{2}$. Punjab is the leading province of Pakistan in respect of population and agriculture. According to physiography, Punjab can be divided into three major regions, such as northern mountains, Potwar Plateau, and the Upper Indus Plain. The general slope of the land is from northeast to southwest. There is also a mountain plateau known as Potohar in the northwest and the southeast, a Cholistan desert. The province's landscape is alluvial plains of Indus rivers with their tributaries, i.e., Jhelum, Chenab, Ravi, and Sutlej. Most of the study area comprises of level plain formed by the Indus River and its eastern tributaries. From the perspective of the climatology of the Punjab province, it is a region with less rainfall and high temperature. The climate is arid to semi-arid, with significant spatio-temporal variations in climatic parameters. There are two major cropping seasons in Punjab, Pakistan. The Kharif season and Rabi season. The Kharif period starts from April to June and ends in October to November, and the Rabi period starts from October to November and ends in March to April. The rice is the major crop of the Kharif season (Bokhari et al., 2017).

The data used in this investigation are the monthly mean temperature, maximum temperature, minimum temperature, and rainfall obtained from the Pakistan Meteorological Department (PMD), including the 19 stations for 1989-2018. The 12 stations were included in Central Punjab, four stations in the Southern Punjab, and the remaining three stations are in Western Punjab. The data of rice production is obtained from Agriculture Statistics of Punjab, Pakistan. For further analysis, the rice crop data is converted into yield ( $\mathrm{Kg} / \mathrm{Hectares})$. The rice crop production is the total cultivated area, and yield is the production per hectare (kilograms).

To make the analysis reliable, adopt the Punjab regions suggested by Wilder (1999). Abbas et al. (2021) further classified these regions on the climate based. The author divided the Punjab province into the Northern, Central, Southern and the Western regions (Fig. 1) based on; Geographical boundaries, official district and division borders, regional economic differences, variations in irrigation, cropping patterns, differences in farm size, land tenure patterns in each region. Several studies have attempted to describe the classification of the Punjab regions (Gazdar, 1999; Malik's 1991 \& 2005). Malik (2005) described classification that limits agronomic regions; however, this classification restricts cropping patterns.

Furthermore, Gazdar's classification of the Punjab province gives more attention to the use of the administrative boundaries for defining and explaining the sub-provincial regions and gives less attention to the canal irrigation system. While the Punjab province considers a wider range of factors based on the geographical boundaries. The climate of the classified regions is predominately dry with high variability in the rainfall, which shifts from east to west and north to south (Cheema et al. 2013; Hanif et al. 2010; Abbas et al., 2016b Abbas et al., 2021a).

The Northern region of the Punjab province gets more precipitation during the whole year than the Southern region. The Southern westerly monsoon rainfall (July to August) is crucial during the summer season to reduce moisture stress in the western region and improve agricultural activity. Whereas, due to western disturbances in Northern Punjab, the rainfall in the winter season (December to March) has also become very helpful for the growth of the Rabi crops. The areas lie in that region where westerly and the easterlies weather system have more interface probability. This region is always under the threat of extreme events of rainfall that may cause torrential rains (Pakistan Meteorological Department, 2010; Abbas and Mayo, 2021c). Figure 2 indicates the various patterns of land use in the Punjab, Pakistan.

Figure 3 indicates the precipitation in the annual and Rabi season for the period of 1979-2020. The Southern region depends on the rainfall of the summer monsoon season that brings a high quantity of rainfall, but it is not enough to fulfill the agricultural sector's requirements. However, this region is mostly dependent on the river, canals, and well irrigation sources. In the southern region of the Punjab, proper irrigation is necessary to carry out agricultural activities. While the northern region, particularly Potohar region, is a rain-fed area, and crops can be full-grown under complementary irrigations (Cheema et al. 2013; Abbas et al., 2018; Abbas et al., 2016a; Abbas and Kousar, 2021d). The climate pattern for both Central and Southern Punjab is different. The central region is wetter, and the southern region is a little drier than the Central. The onset of the southwest monsoon is predicted to Punjab in June to Sialkot or Lahore (Pakistan Meteorological Department, 2018).

\section{Data And Analysis}

This study developed four models to check the effect of wind, temperature, total, irrigated and un-irrigated area, and rainfall on wheat production. Models one and two are developed to have insights about food production in central and northern Punjab, while models 3 and 4 provide information about food production in western and southern Punjab. This study utilized wheat production (WPRD) as a dependent variable while Wheat cultivated area (WCLA), Mean temperature (TMEAN), Maximum temperature (TMAX), Minimum temperature (TMIN), Wind speed (WND), Rainfall (RNF), Total area sown (TSA), Total unirrigated area (TUIRA), Total irrigated area (TIRA) is used as independent variables. The data of mean, maximum, minimum temperature, wind speed, and rainfall is obtained from Pakistan Meteorological Department (PMD) from 1979 to 2020. Moreover, this data was taken from nineteen weather stations in Punjab; twelve are in Central Punjab, four are in Southern Punjab, and the remaining three weather stations are in Western Punjab. The data of wheat production, the total area, sown, total irrigated, and the irrigated area is obtained from Crop Reporting Service Punjab, Agriculture Department Pakistan. The detailed description of variables and data sources is reported in Table 1. 
Table 1

Variable Description and its sources

\begin{tabular}{|llll|}
\hline Variables & Variable Name & Description/definition & Data Sources \\
\hline WPRD & Wheat Production & Annual production $(000$ tons $)$ & Crop Reporting Service (CRS), Punjab Pakistan \\
\hline WCLA & Cultivated area & Annual production $(000$ tons $)$ & Crop Reporting Service (CRS), Punjab Pakistan \\
\hline TMEAN & Average temperature & Average Rabi temperature $\left({ }^{\circ} \mathrm{C}\right)$ & Pakistan Meteorological Department (PMD) Lahore \\
\hline TMAX & Maximum temperature & Average Rabi temperature $\left({ }^{\circ} \mathrm{C}\right)$ & Pakistan Meteorological Department (PMD) Lahore \\
\hline TMIN & Minimum temperature & Average Rabi temperature $\left({ }^{\circ} \mathrm{C}\right)$ & Pakistan Meteorological Department (PMD) Lahore \\
\hline WND & Wind speed & Wind speed in Rabi $\left(\mathrm{ms}^{-}{ }^{-1}\right)$ & Pakistan Meteorological Department (PMD) Lahore \\
\hline RNF & Rainfall & Rainfall in Rabi season $(\mathrm{mm})$ & Pakistan Meteorological Department (PMD) Lahore \\
\hline TSA & Total area sown & Total crops area (ha) & Crop Reporting Service (CRS), Punjab Pakistan \\
\hline TUIRA & Total un-irrigated area & Un irrigated area (ha) & Crop Reporting Service (CRS), Punjab Pakistan \\
\hline TIRA & Total irrigated area & Irrigated area (ha) & Crop Reporting Service (CRS), Punjab Pakistan \\
\hline
\end{tabular}

To obtain empirical results, the following ARDL model is adopted, and all variables are utilized in natural log form.

Simple Linear Model

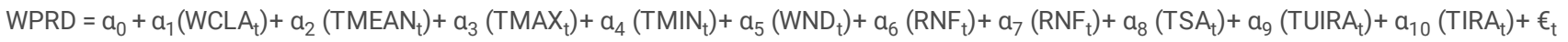

ARDL Specified Models

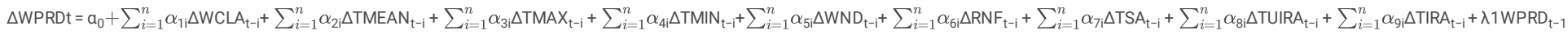
$+\lambda_{2}$ WCLA $_{\mathrm{t}-1}+\lambda_{3}$ TMEAN $_{\mathrm{t}-1}+\lambda_{4}$ TMAX $_{\mathrm{t}-1}+\lambda_{5} \mathrm{TMIN}_{\mathrm{t}-1}+\lambda_{6} \mathrm{WND}_{\mathrm{t}-1}+\lambda_{7} \mathrm{RNF}_{\mathrm{t}-1}+\lambda_{8} \mathrm{TSA}_{\mathrm{t}-1}+\lambda_{9}$ TUIRA $_{\mathrm{t}-1}+\lambda_{10}$ TIRA $_{\mathrm{t}-1}+\epsilon_{\mathrm{t}}$

\section{Empirical Results}

\section{Descriptive Statistics}

Analysis of the study begins with descriptive statists. It summarizes the large pool of data systematically. Results of the descriptive statistics are reported in Table 2. The table shows the mean, median, and standard deviation of the data and the minimum and maximum values of the chosen variables for the selected four regions (i.e., Central, Northern, Western, and Southern). The results of the Jarque-Bera test are also reported in the table. The insignificant probability value of Jarque-Bera indicates the normal distribution of the data.

\section{Unit Root Test}

Table 3 report the results of the ADF and PP unit root test, which has been applied to test the stationarity properties and order or integration of the modeled variables. Both tests are applied for two cases, i.e., with intercept and with the trend and intercept for the selected four regions. Results imply that the modeled variables of the selected four regions are integrated into the mix order, i.e., some variables are stationary at a level. In contrast, others are stationary at the first difference by rejecting the null hypothesis of non-stationary series at $5 \%$ and $1 \%$ significance levels. The Jarque-Bera values indicates the data was normalized. There was no problem of data homogeneity in the data sets for run the functions. 
Table 2

Descriptive Statistic

\begin{tabular}{|c|c|c|c|c|c|c|c|c|}
\hline Variables & Regions & Mean & Median & Maximum & Minimum & Std. Dev. & Jarque-Bera & Probability \\
\hline \multirow[t]{4}{*}{ WPRD } & Central & 8.982 & 9.0733 & 9.2944 & 8.3987 & 0.2705 & 3.9672 & 0.1375 \\
\hline & Northern & 6.138 & 6.1717 & 6.5311 & 5.3552 & 0.2632 & 1.2302 & 0.1060 \\
\hline & Western & 7.251 & 7.4100 & 7.7008 & 6.2168 & 0.4370 & 5.7897 & 0.1553 \\
\hline & Southern & 7.846 & 7.8886 & 8.2116 & 7.3479 & 0.2758 & 3.9207 & 0.1408 \\
\hline \multirow[t]{4}{*}{ WCLA } & Central & 8.118 & 8.1034 & 8.2327 & 7.9986 & 0.0763 & 3.8358 & 0.1469 \\
\hline & Northern & 5.841 & 5.8318 & 6.2936 & 5.61487 & 0.1174 & 1.6564 & 0.1000 \\
\hline & Western & 6.484 & 6.5525 & 6.7175 & 6.0495 & 0.2259 & 1.3260 & 0.2422 \\
\hline & Southern & 6.969 & 6.9768 & 7.0886 & 6.8404 & 0.0677 & 1.9145 & 0.3839 \\
\hline \multirow[t]{4}{*}{ TMEAN } & Central & 2.928 & 2.9326 & 2.9858 & 2.8300 & 0.0311 & 2.4372 & 0.1659 \\
\hline & Northern & 2.863 & 2.8634 & 2.9644 & 2.7715 & 0.0438 & 0.0984 & 0.9519 \\
\hline & Western & 2.908 & 2.9051 & 3.0182 & 2.8269 & 0.0389 & 1.5339 & 0.4644 \\
\hline & Southern & 2.945 & 2.9519 & 3.0470 & 2.6791 & 0.0558 & 2.3804 & 0.1000 \\
\hline \multirow[t]{4}{*}{ TMAX } & Central & 3.229 & 3.2268 & 3.3099 & 3.1973 & 0.0196 & 0.5204 & 0.1000 \\
\hline & Northern & 3.229 & 3.2317 & 3.2976 & 3.1591 & 0.0333 & 1.0649 & 0.5871 \\
\hline & Western & 3.251 & 3.2498 & 3.3077 & 3.2065 & 0.0220 & 1.4243 & 0.4905 \\
\hline & Southern & 3.293 & 3.2900 & 3.3750 & 3.2381 & 0.0306 & 1.0447 & 0.5931 \\
\hline \multirow[t]{4}{*}{ TMIN } & Central & 2.510 & 2.5106 & 2.5888 & 2.4150 & 0.0414 & 0.1119 & 0.9455 \\
\hline & Northern & 2.276 & 2.2950 & 2.4807 & 2.0149 & 0.1035 & 0.6620 & 0.7181 \\
\hline & Western & 2.380 & 2.3892 & 2.5700 & 2.1810 & 0.0887 & 0.2597 & 0.8782 \\
\hline & Southern & 2.416 & 2.4268 & 2.5556 & 1.9606 & 0.0979 & 194.53 & 0.1000 \\
\hline \multirow[t]{4}{*}{ WND } & Central & -0.196 & -0.1924 & 0.3123 & -0.8754 & 0.2699 & 1.9692 & 0.3735 \\
\hline & Northern & -0.468 & -0.4849 & 0.6061 & -1.4552 & 0.4471 & 0.2946 & 0.8630 \\
\hline & Western & 0.419 & 0.4448 & 0.7143 & -0.3298 & 0.2088 & 1.4212 & 0.3002 \\
\hline & Southern & 0.865 & 0.9162 & 2.9159 & 0.0488 & 0.4349 & 2.0952 & 0.1000 \\
\hline \multirow[t]{4}{*}{ RNF } & Central & 5.320 & 5.3122 & 5.9801 & 4.39829 & 0.3585 & 2.1764 & 0.3369 \\
\hline & Northern & 5.259 & 5.2105 & 6.0665 & 4.59510 & 0.3810 & 1.8955 & 0.3879 \\
\hline & Western & 5.0712 & 5.1150 & 5.8074 & 4.25959 & 0.3892 & 2.1627 & 0.3391 \\
\hline & Southern & 3.833 & 3.7716 & 4.6011 & 2.89792 & 0.4578 & 0.8656 & 0.6486 \\
\hline \multirow[t]{4}{*}{ TSA } & Central & 15.707 & 15.7114 & 15.8039 & 15.6243 & 0.0449 & 1.7378 & 0.4194 \\
\hline & Northern & 11.515 & 11.5050 & 11.6345 & 11.4520 & 0.0378 & 2.3749 & 0.1092 \\
\hline & Western & 13.579 & 13.5537 & 13.7442 & 13.4777 & 0.0711 & 2.8308 & 0.2428 \\
\hline & Southern & 14.781 & 14.7806 & 14.8215 & 14.4013 & 0.0196 & 1.0755 & 0.5840 \\
\hline \multirow[t]{4}{*}{ TUIRA } & Central & 12.461 & 12.4625 & 12.6189 & 12.1952 & 0.0973 & 1.2777 & 0.5278 \\
\hline & Northern & 11.198 & 11.2056 & 11.3354 & 11.0542 & 0.0583 & 0.4246 & 0.8087 \\
\hline & Western & 11.538 & 11.5593 & 11.7598 & 11.3286 & 0.1073 & 1.7297 & 0.4210 \\
\hline & Southern & 10.089 & 10.1960 & 10.8382 & 8.47886 & 0.4665 & 1.2673 & 0.1000 \\
\hline \multirow[t]{4}{*}{ TIRA } & Central & 15.669 & 15.6730 & 15.7670 & 15.5940 & 0.0410 & 1.0880 & 0.5804 \\
\hline & Northern & 10.189 & 10.2371 & 10.5178 & 9.89963 & 0.1933 & 4.3404 & 0.1141 \\
\hline & Western & 13.438 & 13.4134 & 13.6507 & 13.2970 & 0.0816 & 3.5973 & 0.1655 \\
\hline & Southern & 14.771 & 14.7712 & 14.0594 & 14.7347 & 0.0183 & 1.7930 & 0.4079 \\
\hline
\end{tabular}




\begin{tabular}{|c|c|c|c|c|c|c|}
\hline & & & Level & & First difference & \\
\hline \multirow[t]{6}{*}{ Variables } & Regions & Test-statistic & With intercept & With intercept and trend & With intercept & With intercept and trend \\
\hline & Central & $A D F$ & -1.3821 & -1.8831 & $-11.1816^{\star \star \star}$ & $-11.178^{\star \star \star}$ \\
\hline & Central & PP & -1.9028 & -2.1537 & 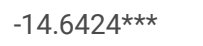 & $-19.787^{\star \star \star}$ \\
\hline & Northern & ADF & $-4.8991^{\star \star \star}$ & 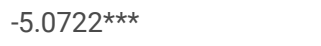 & 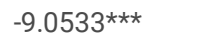 & $-8.9689 * \star \star$ \\
\hline & Northern & PP & $-4.6942^{\star * *}$ & $-5.4847 \star \star \star$ & $-16.9609 * \star \star$ & $-17.702^{\star \star \star}$ \\
\hline & Western & $A D F$ & -2.2003 & -1.8042 & $-8.3506 * \star \star$ & $-8.443^{\star \star \star}$ \\
\hline \multirow[t]{6}{*}{ WPRD } & Western & PP & -0.7274 & -2.9834 & $-9.9271 * \star *$ & $-35.154^{\star \star \star}$ \\
\hline & Southern & ADF & -0.6448 & -2.9146 & $-12.0885^{\star \star \star}$ & $-11.925^{\star \star \star}$ \\
\hline & Southern & PP & -1.0439 & -2.1695 & $-14.6551^{\star \star \star}$ & $-14.448^{\star \star \star}$ \\
\hline & Central & $A D F$ & -1.3342 & -2.8112 & 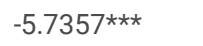 & $-5.715^{\star \star \star}$ \\
\hline & Central & PP & -1.0887 & -2.9223 & $-8.0292^{\star \star \star}$ & $-7.835^{\star \star \star}$ \\
\hline & Northern & $A D F$ & $-4.829 * \star \star$ & $-5.7708^{* \star *}$ & $-11.0077^{\star \star \star \star}$ & $-11.052^{\star \star *}$ \\
\hline \multirow[t]{10}{*}{ WCLA } & Northern & PP & $-5.1036^{* \star \star}$ & $-5.8781^{\star \star \star}$ & $-11.6844^{\star \star \star}$ & $-11.816^{\star \star \star}$ \\
\hline & Western & $A D F$ & -2.1123 & -0.9146 & $-5.5714^{* * *}$ & $-5.974 * \star \star$ \\
\hline & Western & PP & -2.2293 & -0.8026 & -5.5546 *** & $-6.9046^{\star \star \star}$ \\
\hline & Southern & $A D F$ & -2.6999 & -2.2231 & $-5.7208^{\star \star \star}$ & $-5.5877^{\star \star \star}$ \\
\hline & Southern & PP & -2.5649 & -2.9842 & $-14.2962^{\star \star \star}$ & $-16.809 * \star \star$ \\
\hline & Central & $A D F$ & $-4.4715^{\star \star \star}$ & $-4.4353^{\star \star \star}$ & $-9.0611 \star \star \star *$ & $-8.939 * * *$ \\
\hline & Central & PP & $-4.4768^{* * *}$ & $-4.4309 * * *$ & $-13.9541^{\star \star \star}$ & $-13.569 * \star *$ \\
\hline & Northern & $A D F$ & $-3.3281^{\star \star}$ & $-3.1422^{\star \star}$ & $-11.1813^{\star \star \star}$ & $-11.034^{\star \star \star}$ \\
\hline & Northern & PP & $-4.2049 * * *$ & $-5.3161^{\star \star *}$ & $-13.7438^{\star \star \star}$ & $-13.572^{\star \star \star}$ \\
\hline & Western & $A D F$ & -2.0938 & -2.2897 & $-10.8426 * \star \star$ & $-10.527^{\star \star \star}$ \\
\hline \multirow[t]{7}{*}{ TMEAN } & Western & PP & $-3.4751 * \star$ & $-4.2686^{\star * *}$ & $-10.8906^{\star \star \star}$ & $-10.795^{\star \star \star}$ \\
\hline & Southern & $A D F$ & $-5.3341^{\star \star *}$ & $-6.2205^{\star \star \star}$ & $-7.6436^{\star \star \star}$ & $-7.533^{\star \star \star}$ \\
\hline & Southern & PP & $-5.5719 * \star \star$ & $-6.2312^{\star \star \star}$ & $-34.0501^{* * *}$ & $-33.621^{\star \star \star}$ \\
\hline & Central & $A D F$ & $-6.6335^{\star \star \star}$ & $-6.4643^{\star \star \star}$ & $-10.1758^{\star \star \star}$ & $-15.521^{\star \star \star}$ \\
\hline & Central & PP & $-7.1764^{\star \star *}$ & $-6.9032^{\star \star \star}$ & 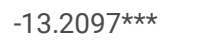 & $-12.831^{\star \star \star}$ \\
\hline & Northern & $A D F$ & $-5.8379 * * *$ & $-6.0752^{\star \star *}$ & $-10.8227^{\star \star \star}$ & $-10.678 * \star \star$ \\
\hline & Northern & PP & $-5.8362^{\star * *}$ & $-6.0797 * \star \star$ & $-20.4506^{\star \star \star}$ & $-20.105^{\star \star \star}$ \\
\hline \multirow[t]{7}{*}{ TMAX } & Western & $A D F$ & $-4.8889 * * *$ & $-4.7943^{\star \star *}$ & $-8.5256^{\star \star \star}$ & 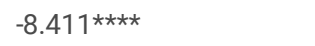 \\
\hline & Western & PP & $-4.9579 * * *$ & $-4.8733^{\star \star \star}$ & $-8.3985^{\star \star \star}$ & $-8.8453^{\star \star \star}$ \\
\hline & Southern & $A D F$ & $-4.6183^{* * *}$ & $-4.8283^{\star * *}$ & $-9.7462^{\star \star \star}$ & $-9.6220 * \star *$ \\
\hline & Southern & PP & $-4.6892^{\star \star \star}$ & $-4.9024^{\star \star \star}$ & $-14.8795^{\star \star \star}$ & $-14.909 * \star \star$ \\
\hline & Central & $\mathrm{ADF}$ & $-3.4796^{\star \star}$ & $-3.4331^{\star \star}$ & $-7.3862^{\star \star \star}$ & $-7.291^{\star \star \star}$ \\
\hline & Central & PP & $-3.4796^{\star \star}$ & $-3.4331 * \star$ & $-9.5739 * \star \star$ & $-9.508^{\star \star \star}$ \\
\hline & Northern & $A D F$ & -1.5835 & -2.0912 & $-10.3672^{\star \star \star}$ & $-10.239 * \star \star$ \\
\hline \multirow[t]{5}{*}{ TMIN } & Northern & PP & -2.4828 & -2.7062 & $-10.3672^{\star \star *}$ & $-10.239 * \star \star$ \\
\hline & Western & $A D F$ & -1.4671 & -2.1895 & $-9.6849 * * *$ & $-9.5587 * \star *$ \\
\hline & Western & PP & -2.0214 & -1.1993 & 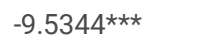 & $-9.0036 * \star \star$ \\
\hline & Southern & $A D F$ & $-5.2472^{\star \star *}$ & $-6.2952^{\star \star \star}$ & 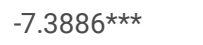 & $-7.2839 * \star \star$ \\
\hline & Southern & PP & $-5.3694^{* * *}$ & $-6.2952^{\star \star *}$ & $-38.0372^{\star \star \star}$ & $-37.167 * \star \star$ \\
\hline
\end{tabular}




\begin{tabular}{|c|c|c|c|c|c|c|}
\hline & \multirow[b]{2}{*}{ Central } & \multirow[b]{2}{*}{ ADF } & \multicolumn{2}{|l|}{ Level } & \multicolumn{2}{|c|}{ First difference } \\
\hline & & & $-4.2225^{\star \star \star}$ & $-4.5738^{\star \star \star}$ & $-8.7339 * \star \star$ & $-8.6452^{\star \star \star}$ \\
\hline & Central & PP & $-3.9923^{\star \star}$ & $-4.3695^{\star \star}$ & $-12.7148 * \star \star$ & 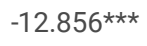 \\
\hline & Northern & ADF & $-3.8485^{\star \star}$ & $-3.8837 * \star$ & $-8.6495^{\star \star \star}$ & $-8.6730 * \star *$ \\
\hline \multirow[t]{8}{*}{ WND } & Northern & PP & $-3.8224^{\star *}$ & $-3.8269 * \star$ & $-12.4226^{\star \star \star}$ & $-20.579 \star \star * *$ \\
\hline & Western & ADF & $-4.8923^{\star \star \star}$ & $-4.9541 * \star \star$ & $-6.4802^{\star \star \star}$ & $-6.3181 * * *$ \\
\hline & Western & PP & $-4.7553^{\star \star \star}$ & $-4.7975^{\star \star \star}$ & $-19.6361^{\star \star \star}$ & $-19.918 * \star \star *$ \\
\hline & Southern & ADF & 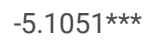 & $-5.1393^{\star \star \star}$ & $-9.2076^{\star \star \star}$ & $-9.3743^{\star \star *}$ \\
\hline & Southern & PP & $-5.1051^{\star \star \star}$ & 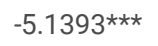 & 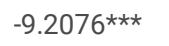 & $-9.3743^{\star \star \star}$ \\
\hline & Central & ADF & $-3.7502^{\star \star}$ & 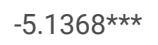 & $-9.6399 \star \star \star$ & $-9.5146 * \star *$ \\
\hline & Central & PP & $-3.6505^{\star \star}$ & $-4.1985^{\star \star \star}$ & $-26.7461^{\star \star \star}$ & $-26.860 * \star \star *$ \\
\hline & Northern & ADF & $-5.3712^{\star \star \star}$ & $-5.3102^{\star \star \star}$ & $-10.8611^{\star \star \star \star}$ & 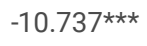 \\
\hline \multirow[t]{7}{*}{ RNF } & Northern & PP & $-5.4165^{\star \star \star}$ & $-5.3579 * \star \star$ & $-20.2389 * \star \star$ & $-21.045^{\star \star \star}$ \\
\hline & Western & ADF & $-3.5375^{\star \star \star}$ & $-4.7352^{\star \star \star}$ & $-9.3171 * \star \star$ & $-9.2266^{\star \star \star}$ \\
\hline & Western & PP & $-3.4876^{\star \star}$ & $-4.3384 * \star \star$ & $-12.7394 * \star \star$ & $-12.569 * \star *$ \\
\hline & Southern & ADF & $-3.7844^{\star \star}$ & $-3.7408^{\star \star}$ & 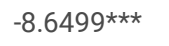 & $-8.5384^{\star \star \star}$ \\
\hline & Southern & PP & $-4.3525^{\star \star \star}$ & 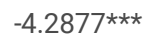 & $-12.9816^{\star \star \star}$ & $-12.870 * \star \star$ \\
\hline & Central & ADF & -2.6694 & -2.8255 & $-5.1242^{\star \star \star}$ & $-5.0578 * \star \star$ \\
\hline & Central & PP & -1.9114 & -2.1942 & $-7.0126^{\star \star \star}$ & $-6.9246 * \star *$ \\
\hline \multirow[t]{10}{*}{ TSA } & Northern & ADF & -4.0679 & -4.0233 & $-7.5588^{\star \star \star}$ & $-7.4543^{\star \star \star}$ \\
\hline & Northern & PP & -4.3744 & -4.0027 & 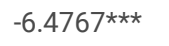 & $-6.2361 * \star \star$ \\
\hline & Western & ADF & -2.5913 & -2.515 & $-6.9853^{\star \star \star}$ & $-6.9463^{* \star *}$ \\
\hline & Western & PP & $-3.0655^{\star \star}$ & $-3.0944^{\star *}$ & 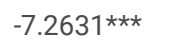 & $-7.1665^{\star \star \star}$ \\
\hline & Southern & ADF & $-3.8954^{\star *}$ & $-4.0068^{\star \star \star}$ & $-8.2606^{\star \star \star}$ & $-8.1484^{\star \star \star}$ \\
\hline & Southern & PP & $-3.8896^{\star *}$ & $-4.0176^{\star \star \star}$ & $-18.726^{\star \star \star}$ & $-18.118^{\star \star *}$ \\
\hline & Central & ADF & -2.8631 & -2.808 & $-6.4497 * \star \star$ & $-6.3830 * \star \star *$ \\
\hline & Central & PP & -2.8844 & -2.8381 & $-6.7505^{\star \star \star}$ & $-6.9261 * \star \star *$ \\
\hline & Northern & ADF & $-3.6534^{\star \star}$ & $-3.8456 * \star$ & $-5.3645^{\star \star \star}$ & $-5.8354 * \star *$ \\
\hline & Northern & PP & $-3.1291 \star *$ & $-3.2139 \star \star$ & 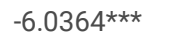 & $-5.9539 * \star \star$ \\
\hline \multirow[t]{9}{*}{ TUIRA } & Western & ADF & $-3.0655^{\star \star}$ & $-3.0944 * \star$ & $-7.2631^{\star \star \star \star}$ & $-7.1665^{\star \star \star}$ \\
\hline & Western & PP & $-3.0857 \star \star$ & $-3.1314^{\star \star}$ & $-7.2731 * \star \star$ & $-7.1756^{\star \star \star}$ \\
\hline & Southern & ADF & $-5.9451 \star \star \star *$ & $-6.2714^{\star \star \star}$ & $-7.5734^{\star \star \star}$ & $-7.4691 * \star *$ \\
\hline & Southern & PP & $-6.0479 \star \star \star *$ & $-6.3131^{\star \star \star}$ & $-13.0831^{\star \star \star}$ & $-12.905^{\star \star \star}$ \\
\hline & Central & ADF & -2.2814 & -2.3817 & 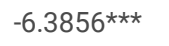 & $-6.3005^{\star \star \star}$ \\
\hline & Central & PP & -1.7344 & -2.3743 & $-5.8475^{\star \star \star}$ & 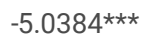 \\
\hline & Northern & ADF & -2.2837 & -2.3091 & $-6.9684 * \star \star$ & $-6.8805^{\star \star \star}$ \\
\hline & Northern & PP & -2.2254 & -2.2628 & $-6.9762^{\star \star \star}$ & $-6.8874 * \star \star$ \\
\hline & Western & ADF & -2.4948 & -2.4109 & $-7.3705^{\star \star \star}$ & $-7.2404^{\star \star \star}$ \\
\hline \multirow[t]{3}{*}{ TIRA } & Western & PP & -2.4623 & -2.3767 & $-7.1105^{\star \star \star}$ & $-7.2385^{\star \star \star}$ \\
\hline & Southern & ADF & $-4.0873^{\star \star \star}$ & $-4.1744^{\star \star \star}$ & $-8.6293^{\star \star \star}$ & $-8.5143^{\star \star *}$ \\
\hline & Southern & PP & $-4.0373^{\star \star \star}$ & $-4.1449 * \star \star$ & $-21.3582^{\star \star \star}$ & $-21.336^{\star \star \star}$ \\
\hline
\end{tabular}


Time series data usually suffer from auto/serial correlation, heteroscedasticity, the problem of multicollinearity, and the problem of model misspecification, which, if not detected, provide indefinite results. The study, therefore, applies some diagnostic tests to detect these problems for the case of selected regions. The study applies Breusch-Godfrey Serial Correlation to detect serial correlation, Breusch-Pagan-Godfry HSK is applied to test the problem of heteroscedasticity, and Ramsey Reset test is applied to check the model's specification. The results of Breusch-Godfrey Serial Correlation, Breusch-PaganGodfry HSK, Ramsey Reset test and correlation are reported in Table 4. The insignificant p-values of Breusch-Godfrey Serial Correlation and Breusch-PaganGodfry HSK (see Table 4) indicate that the problem of serial correlation and heteroscedasticity does not exist in our proposed models. The insignificant pvalues of the Ramsey Reset test (see Table 4) indicate that the proposed models of the study are correctly specified. Results of Table 4 show that the problem of multicollinearity does not exist in the data because all coefficient values of correlation among the modeled variables are less than 0.5 . 


\begin{tabular}{|c|c|c|c|c|c|c|c|c|c|c|c|}
\hline \multicolumn{12}{|c|}{ Panel A: Central } \\
\hline & WPRD & WNDS & \multicolumn{2}{|l|}{ WCLA } & TUIRA & TSA & \multicolumn{2}{|l|}{ TMIX } & TMEAN & TMAX & \multirow[t]{2}{*}{ RNF } \\
\hline WPRD & 1 & & & & & & & & & & \\
\hline WNDS & 0.328 & 1 & & & & & & & & & \\
\hline WCLA & 0.196 & 0.356 & 1 & & & & & & & & \\
\hline TUIRA & -0.17 & -0.057 & -0.193 & & 1 & & & & & & \\
\hline TSA & -0.25 & -0.173 & -0.292 & & 0.751 & 1 & & & & & \\
\hline TMIX & -0.26 & -0.106 & -0.324 & & 0.107 & 0.079 & 1 & & & & \\
\hline TMEAN & 0.13 & 0.029 & -0.030 & & 0.071 & 0.091 & 0.239 & & 1 & & \\
\hline TMAX & -0.19 & -0.381 & -0.206 & & 0.198 & 0.341 & 0.284 & & 0.201 & 1 & \\
\hline RNF & -0.13 & -0.101 & -0.587 & & 0.207 & 0.362 & -0.057 & & -0.164 & -0.176 & 1 \\
\hline TIRA & -0.22 & -0.150 & -0.264 & & 0.255 & 0.985 & 0.069 & & 0.096 & 0.313 & 0.387 \\
\hline \multicolumn{12}{|c|}{ Panel B: Northern } \\
\hline & WPRD & & WNDS & WCLA & TUIRA & & TSA & TMIX & TMEAN & TMAX & RNF \\
\hline WPRD & 1 & & & & & & & & & & \\
\hline WND & -0.098 & & 1 & & & & & & & & \\
\hline WCLA & 0.329 & & 0.167 & 1 & & & & & & & \\
\hline TSA & 0.031 & & -0.156 & 0.032 & 1 & & & & & & \\
\hline TUIRA & -0.105 & & -0.030 & 0.046 & 0.191 & & 1 & & & & \\
\hline TMIN & 0.129 & & -0.051 & -0.361 & -0.182 & & 0.050 & 1 & & & \\
\hline TMEAN & 0.205 & & -0.008 & -0.283 & -0.354 & & -0.19 & 0.160 & 1 & & \\
\hline TMAX & 0.223 & & 0.049 & -0.084 & -0.421 & & -0.48 & 0.383 & 0.199 & 1 & \\
\hline TIRA & 0.104 & & -0.113 & -0.015 & 0.480 & & -0.38 & -0.224 & -0.107 & 0.088 & 1 \\
\hline RNF & -0.095 & & -0.082 & -0.093 & 0.285 & & 0.446 & 0.095 & -0.211 & -0.296 & -0.151 \\
\hline \multicolumn{12}{|c|}{ Panel C: Western } \\
\hline & WPRD & & WNDS & WCLA & TUIRA & & TSA & TMIX & TMEAN & TMAX & RNF \\
\hline WPRD & 1 & & & & & & & & & & \\
\hline WND & -0.204 & & 1 & & & & & & & & \\
\hline WCLA & 0.163 & & -0.165 & 1 & & & & & & & \\
\hline TSA & -0.103 & & -0.119 & -0.118 & 1 & & & & & & \\
\hline TUIRA & 0.127 & & -0.116 & 0.203 & 0.128 & & 1 & & & & \\
\hline TMIN & 0.755 & & -0.332 & 0.254 & 0.113 & & -0.06 & 1 & & & \\
\hline TMEAN & 0.211 & & -0.335 & 0.119 & 0.104 & & -0.14 & 0.814 & 1 & & \\
\hline TMAX & 0.126 & & -0.162 & 0.171 & 0.255 & & -0.16 & 0.367 & 0.720 & 1 & \\
\hline TIRA & 0.143 & & -0.094 & 0.227 & -0.074 & & 0.27 & -0.088 & -0.165 & -0.214 & 1 \\
\hline RNF & -0.218 & & 0.103 & -0.491 & 0.295 & & 0.25 & -0.310 & -0.386 & -0.155 & 0.161 \\
\hline \multicolumn{12}{|c|}{ Panel D: Southern } \\
\hline & WPRD & & WNDS & WCLA & TUIRA & TSA & TM & & TMEAN & TMAX & RNF \\
\hline WPRD & 1 & & & & & & & & & & \\
\hline WND & 0.199 & & 1 & & & & & & & & \\
\hline WCLA & 0.164 & & 0.244 & 1 & & & & & & & \\
\hline TSA & -0.199 & & -0.086 & -0.129 & 1 & & & & & & \\
\hline
\end{tabular}




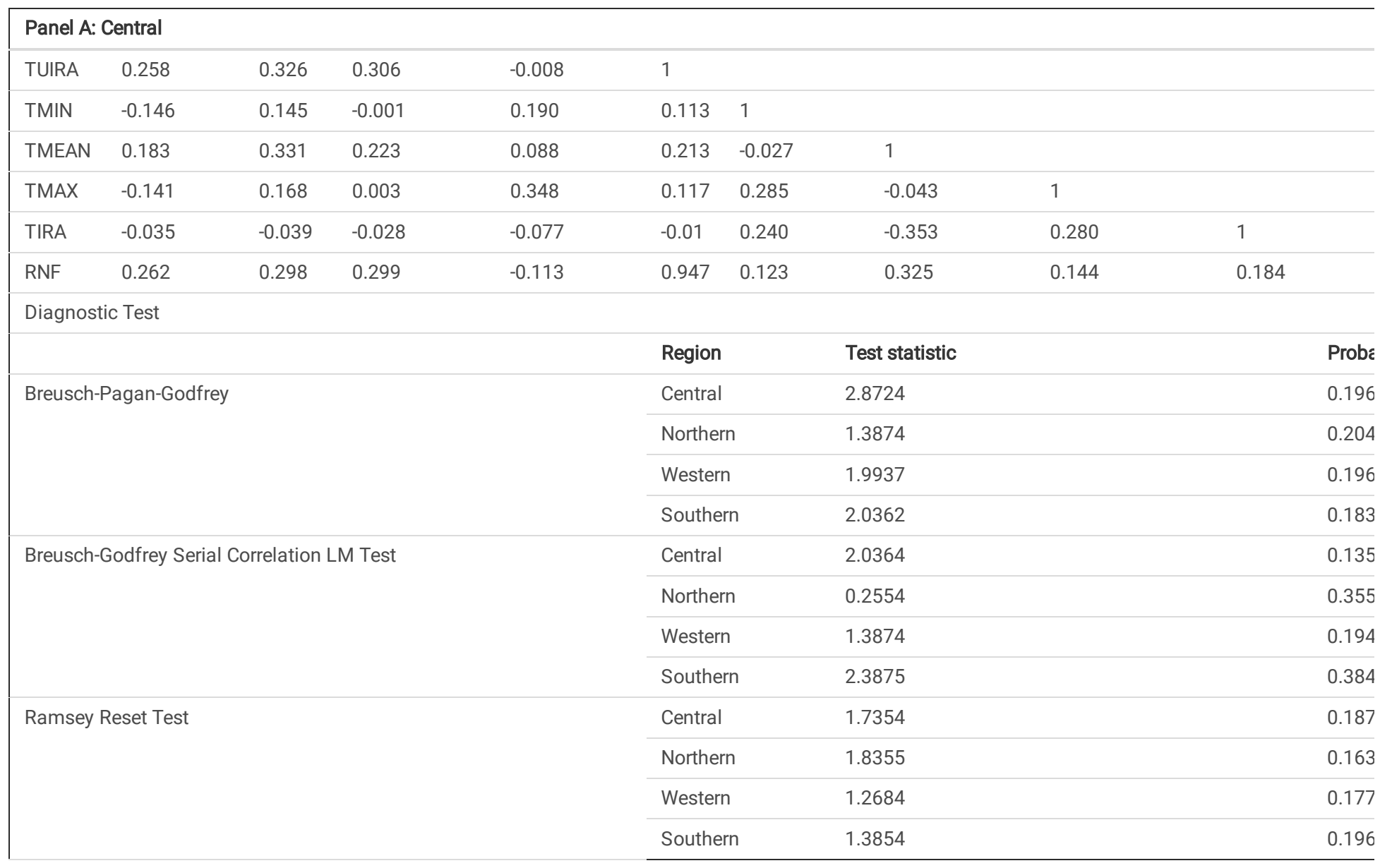

\section{Bounds Cointegration Test}

The present study applies the ARDL approach to estimate the empirical results of the study. The first step of ARDL is to test the cointegrating relation between the selected variables of the study through the bounds cointegration test. The bound test is a pre-condition of applying ARDL, which tells that either the chosen variables of the study are moving together in the long run or not.

Table 5

Bounds Co-integration Test

\begin{tabular}{|lll|}
\hline Region & K & F. Statistics \\
\hline Central Punjab & 9 & 5.7344 \\
\hline Northern Punjab & 9 & 7.9767 \\
\hline Western Punjab & 9 & 4.3644 \\
\hline Southern Punjab & 9 & 7.5143 \\
\hline Significance & Lower Bound & Upper Bound \\
\hline $10 \%$ & 2.05 & 3.02 \\
\hline $5 \%$ & 2.30 & 3.33 \\
\hline $1 \%$ & 2.79 & 3.93 \\
\hline
\end{tabular}

The bound test is having the null hypothesis of "no-cointegration." The rejection of this hypothesis indicates the cointegrating relation among the modeled variables. The results of the bounds cointegration tests are reported in Table 5 . The table shows that the value of $F$ statistics is greater than the upper bound at the significance level of $10 \%, 5 \%$, and $1 \%$ for the selected regions. So here, it is concluded that cointegration exists among the chosen variables and all the variables move together in the long run.

\section{Short-run Effects Of Climate On Wheat}

The results of the short run for the selected four regions in models 1-4 respectively are reported in Table 6. Results depict that in model 1, the value of ECM is -0.6845 , which is negative and highly significant at the level of $1 \%$. This shows that $68.45 \%$ of inconsistency between the long-term and short-term wheat production of the central region is corrected within a year, while the value of ECM is -0.7454 in model 2 . The ECM term indicates that $74.54 \%$ of inconsistency 
between the long-term and short-term wheat production of the Northern region is corrected within a year at a $1 \%$ level of significance. The value of ECM in model 3 is -0.7767 , which is also significant at the level of $1 \%$. The result shows that the speed of adjustment between the short and long-run wheat production for the Western region is $77.67 \%$. However, this value is -0.6967 in model 4 , depicting that the speed of adjustment is $69.67 \%$ for the case of the Southern region.

\section{Long Run Effects Of Climate On Wheat}

\section{Brief model descriptions}

The long-run results of models 1,2,3 and 4 are presented in Table 7. Before moving towards a detailed explanation of the long-run results, this study provides a brief description of the table and its models for the readers so that they can easily understand the results. Table 7 is comprised of four models. Model 1 report the long-run coefficients of the central region, model 2 present the results of Northern region, model 3 and 4 exhibits the long-run coefficients of Western and Southern region, respectively. However, the visual representation of the proposed relationship among the modeled variables is presented in Figs. $1 \mathrm{~A}$, 1B, 1C, and 1D for Central, Northern, Western, and Southern regions, respectively. 
Table 6

Short-run results

\begin{tabular}{|c|c|c|c|c|}
\hline DV: WPRD & Models/Regions & & & \\
\hline Independent Variables & Model 1: Central & Model 2: Northem & Model 3: Western & Model 4: Southern \\
\hline \multirow[t]{2}{*}{ WPRD(-1) } & $-0.8473^{\star \star \star}$ & 0.1311 & 0.0318 & -0.1659 \\
\hline & $(0.0259)$ & $(0.3817)$ & $(0.8794)$ & $(0.2856)$ \\
\hline \multirow[t]{2}{*}{ WCLA } & $2.1061^{\star \star \star}$ & 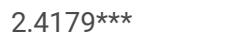 & 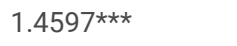 & $0.7449 \star \star$ \\
\hline & $(0.0074)$ & $(0.0000)$ & $(0.0012)$ & $(0.0212)$ \\
\hline \multirow[t]{2}{*}{ WCLA (-1) } & $-1.2662^{\star \star}$ & $-1.1998 * \star$ & 0.3757 & -0.1907 \\
\hline & $(0.0416)$ & $(0.0218)$ & $(0.3474)$ & $(0.5678)$ \\
\hline \multirow[t]{2}{*}{ WCLA (-2) } & -1.1967 & $0.8562^{\star \star \star}$ & 0.3355 & 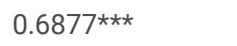 \\
\hline & $(0.1823)$ & $(0.0091)$ & $(0.2341)$ & $(0.0137)$ \\
\hline \multirow[t]{2}{*}{ TMEAN } & 0.4441 & $-4.0384^{\star \star}$ & $0.9647 * \star$ & -0.4416 \\
\hline & $(0.6062)$ & $(0.0481)$ & $(0.0279)$ & $(0.7847)$ \\
\hline \multirow[t]{2}{*}{ TMEAN (-1) } & $1.5137^{\star}$ & $-2.3465^{\star \star}$ & 0.8743 & -1.1645 \\
\hline & $(0.088)$ & $(0.0375)$ & $(0.2532)$ & $(0.6355)$ \\
\hline \multirow[t]{2}{*}{ TMEAN (-2) } & $-2.0796 \star \star \star$ & $-3.3564^{\star}$ & 0.6237 & -1.0354 \\
\hline & $(0.0073)$ & $(0.0765)$ & $(0.3542)$ & $(0.4766)$ \\
\hline \multirow[t]{2}{*}{ TMAX } & $7.24843^{* * *}$ & 4.8313 & 0.6163 & 0.1313 \\
\hline & $(0.006)$ & $(0.4985)$ & $(0.5924)$ & $(0.4362)$ \\
\hline \multirow[t]{2}{*}{$\operatorname{TMAX}(-1)$} & $2.5985^{\star \star \star}$ & 4.25384 & -0.0608 & 0.4047 \\
\hline & $(0.0036)$ & $(0.2433)$ & $(0.9146)$ & $(0.1656)$ \\
\hline \multirow[t]{2}{*}{ TMAX (-2) } & $2.3746^{\star \star}$ & 3.8357 & $2.4804^{\star \star \star}$ & 1.1227 \\
\hline & $(0.0354)$ & (0.1733) & $(0.0015)$ & $(0.7634)$ \\
\hline \multirow[t]{2}{*}{ TMIN } & $-1.5507 \star \star$ & 2.6707 & $-0.4165^{\star \star}$ & 0.4696 ** \\
\hline & (0.0309) & $(0.6402)$ & $(0.0221)$ & $(0.0414)$ \\
\hline \multirow[t]{2}{*}{$\operatorname{TMIN}(-1)$} & $-1.4755^{\star \star}$ & 2.4573 & -0.3754 & 0.8068 \\
\hline & $(0.0353)$ & $(0.5244)$ & $(0.1874)$ & $(0.1874)$ \\
\hline \multirow[t]{2}{*}{ TMIN (-2) } & -1.3758 & 1.9476 & -0.2863 & 0.74555 \\
\hline & $(0.3733)$ & $(0.3584)$ & $(0.1753)$ & $(0.2744)$ \\
\hline \multirow[t]{2}{*}{ WND } & $0.0726^{\star \star}$ & $-0.2781 * \star \star$ & 0.0558 & $-0.0637^{*}$ \\
\hline & $(0.0598)$ & $(0.0009)$ & (0.3995) & $(0.0702)$ \\
\hline \multirow[t]{2}{*}{ WND (-1) } & 0.05674 & $-0.1424^{\star}$ & $0.0595^{\star}$ & $-0.0622^{\star}$ \\
\hline & $(0.2654)$ & $(0.0846)$ & $(0.0808)$ & $(0.0781)$ \\
\hline \multirow[t]{2}{*}{ WND (-2) } & 0.0352 & $-0.12844^{\star \star}$ & $0.0887 * *$ & $-0.04653^{\star *}$ \\
\hline & $(0.1654)$ & $(0.0364)$ & $(0.0456)$ & $(0.0274)$ \\
\hline \multirow[t]{2}{*}{ RNF } & $0.2300 * \star \star$ & $0.1885^{\star \star}$ & 0.0974 & $-0.0761^{\star}$ \\
\hline & $(0.0299)$ & $(0.0346)$ & $(0.4567)$ & $(0.0647)$ \\
\hline \multirow[t]{2}{*}{ RNF (-1) } & $0.3243^{\star \star \star}$ & 0.1104 & 0.0351 & $-0.06418 * \star \star$ \\
\hline & $(0.0079)$ & $(0.1571)$ & $(0.2534)$ & $(0.0092)$ \\
\hline \multirow[t]{2}{*}{ RNF (-2) } & 0.2725 & 0.0873 & $0.0223^{*}$ & -0.03455 \\
\hline & $(0.2656)$ & $(0.1446)$ & $(0.0645)$ & $(0.8734)$ \\
\hline \multirow[t]{2}{*}{ TSA } & 1.4948 & 2.4387 & $-2.9631^{\star \star \star}$ & 0.5023 \\
\hline & $(0.5269)$ & $(0.7472)$ & $(0.0315)$ & $(0.4703)$ \\
\hline
\end{tabular}




\begin{tabular}{|c|c|c|c|c|}
\hline DV: WPRD & Models/Re & & & \\
\hline TSA (-1) & $1.39835^{\star}$ & $2.0624^{\star}$ & -2.48391 & $7.7270^{\star}$ \\
\hline & $(0.0745)$ & $(0.0799)$ & $(0.0364) * \star \star$ & $(0.0723)$ \\
\hline TSA (-2) & 1.2036) & $2.2764^{\star \star}$ & $-3.81934^{\star \star \star}$ & 5.7645 \\
\hline & $(0.0365)$ & $(0.0284)$ & $(0.0153)$ & $(0.9622)$ \\
\hline TUIRA & -0.29484 & -1.3662 & $4.3065^{\star *}$ & -0.0535 \\
\hline & $(0.284)$ & $(0.8047)$ & $(0.0344)$ & $(0.6304)$ \\
\hline TUIRA (-1) & -0.3771 & -10.9396 & $4.2922^{\star \star \star}$ & -0.15471 \\
\hline & $(0.1578)$ & $(0.1106)$ & $(0.0418)$ & $(0.1884)$ \\
\hline TUIRA $(-2)$ & -0.32078 & $-1.7583^{*}$ & $2.8735^{\star \star \star}$ & $-0.13434 *$ \\
\hline & $(0.1796)$ & $(0.0932)$ & $(0.0344)$ & $(0.0745)$ \\
\hline TIRA & $-0.6092^{*}$ & -1.2046 & $2.0818^{\star \star \star}$ & $0.7726^{\star \star \star}$ \\
\hline & $(0.0825)$ & $(0.5508)$ & $(0.034)$ & $(0.0007)$ \\
\hline $\operatorname{TIRA}(-1)$ & -4.31381 & $-3.6737 * \star$ & $2.9878^{\star \star \star}$ & -9.1978 \\
\hline & $(0.4544)$ & $(0.03762)$ & $(0.0377)$ & $(0.9417)$ \\
\hline TIRA (-2) & -0.84789 & -2.3467 & 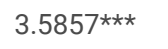 & -7.3645 \\
\hline & $(0.2764)$ & $(0.1344)$ & $(0.0010)$ & $(0.6455)$ \\
\hline ECM & $-0.6845^{\star \star \star}$ & $-0.7454^{\star \star \star}$ & $-0.7767 * \star \star$ & $-0.6967 * \star \star$ \\
\hline & $(0.0000)$ & $(0.0000)$ & $(0.0000)$ & $(0.0000)$ \\
\hline Constant & $3.3344^{\star \star}$ & $4.3655^{\star \star}$ & $2.3645^{\star \star \star}$ & $4.47566^{\star \star}$ \\
\hline & $(0.0354)$ & $(0.0337)$ & $(0.0264)$ & $(0.0265)$ \\
\hline
\end{tabular}

\section{Coefficient Interpretations}

\section{Wheat cultivated area}

The coefficient of WCLA in model 1 is 0.4545 , which is significant at the level of $10 \%$ (i.e., p-value $=0.0734$ ). Result reveals the positive relationship between WCLA and WPRD in the long run and implies that a 1-unit increase in WLCA will increase 0.4545 units of WPRD for the central region. The coefficient of WCLA in model $2(1.3875)$ is significant at the level of $1 \%$ (i.e., p-value $=0.0005$ ) and reveals the positive affiliation between WCLA and WPRD. The result indicates that a 1-unit increase in WCLA tends to increase 1.3875 units of WPRD in the long run for the case northern region. The coefficient of WCLA in model 3 (1.8951) and model 4 (1.0652) is also significant at the level of $1 \%$ (i.e., p-value $=0.0000$ and 0.0094 , respectively). Result states that in the long run, a 1 -unit increase in WCLA will increase 1.8951 units of WPRD in the western region while 1.0652 units of WPRD in the southern region. The overall results suggest the positive impact of WCLA on WPRD in the long run, and thus, (H1) is supported for all the selected regions.

\section{Mean temperature}

In model 1, the coefficient of TMEAN is negative and significant at the level of $5 \%$ (i.e., $\beta=-1.0653$; $p$-value $=0.0255$ ), which shows the negative relationship between TMEAN and WPRD. The result indicates that a 1-unit increase in TMEAN will reduce 1.0653 units of WPRD for the case Central region in the long run. The coefficient of TMEAN in model $2(-1.1583)$ is also negative and significant at the level of $1 \%$. The result implies that a 1-unit increase in TMEAN will reduce 1.1583 units of WPRD for the case of the northern region. The coefficient of TMEAN in model 3 (-0.9964) also reveals the negative long-run impact of TMEAN on WPRD at $5 \%$ significant level (i.e., p-value $=0.0341$ ). Result states that a 1 -unit of increase in TMEAN will reduce 0.9964 units of WPRD for the case of the Western region.

Similarly, in the southern region, there also exists a negative relationship between TMEAN and WPRD. The result shows that a 1-unit increase in TMEAN will result from a decrease of 1.3776 units in WPRD in the long run. The overall results suggest the positive impact of TMEAN on WPRD in the long run, and thus, (H2) is supported for all the selected regions.

\section{Maximum temperature}

The coefficient of TMAX in model 1 (-3.3287) reveals the significant and negative association between TMAX and WPRD at the level of 1\% (i.e., p-value = 0.0006). The result portrays that a 1-unit increase in TMAX will reduce 3.3287 units of WPRD for the case of the Central region. While, in model 2 , the coefficient of TMAX (1.0711) is insignificant, depicting no long-run relationship between TMAX and WPRD for the case of the northern region. In models 3 and 4, the coefficient of TMAX is -3.1324 and - 1.4222, respectively. The result shows that in the long run, a 1-unit increase in TMAX will reduce 3.1324 units of WPRD in the western region, while 1.4222 units of WPRD in the southern region. The overall result indicates the negative role of TMAX on WPRD for the case of the Central, Western and Southern regions. Hence, $(\mathrm{H} 3)$ is rejected for the case of the Northern region. 


\section{Minimum temperature}

The coefficient of TMIN is negative and significant at level of $5 \%$ in model $1(\beta=-1.8413, p$-value $=0.0359)$, at the level of $1 \%$ in model $2(\beta=-2.0740, p-v a l u e=$ $0.0045)$ and at the level of $10 \%$ in model $4(\beta=-1.0949$, $p$-value $=0.078)$. the result reveals the negative long-run impact of TMIN on WPRD. The result implies that a 1-unit increase in TMIN will reduce 1.8413 units of WPRD in the Central region, while 2.0740 and 1.0949 units of WPRD in the western and southern regions, respectively. On the contrary, in the Northern region, the coefficient of TMIN (-2.0740) is insignificant and reveals no LR association between TMIN and WPRD for the Western region. The overall result portrays the negative impact of TMIN on WPRD for the Central, Northern and Southern regions. Therefore, (H4) is rejected for the case of the Western region.

\section{Wind speed}

The coefficient of WND in model 1 is -0.2176 , which is significant at the level of $5 \%$ (i.e., $p$-value $=0.0269$ ). The result portrays the negative effect of WND on WPRD in the long run. Findings indicated that a 1-unit increase in WND would reduce 0.2176 units of WPRD for the central region. However, in model 2 , the coefficient of WND (1.4849) is significant, which shows that WND does not have any significant effects on WPRD for the case of the Northern region. While, in models 3 , and 4 WND has a significant negative impact on WPRD in the long run. The result depicts that 1 -unit of increase in WND brings - 0.2109 units of reduction in WPRD for the case of the Western region, while -0.1085 units of reduction in Southern region at the level of $10 \%$ and $1 \%$ respectively. The overall results support (H5) for the case of the Central, Western and Southern regions.

\section{Rainfall}

The coefficient of RNF is insignificant in model 1 (i.e., $\beta=0.3000$, $p$-value $=0.1254$ ), and model $3(\beta=0.1363$, $p$-value $=0.4548$ ), which depicts that in the long run, any change in RNF brings no change in WPRD for the case of Central and Western region. On the contrary, the coefficient of RNF is significant at the level of $5 \%$ in model 2 (i.e., $\beta=-0.3441$, $p$-value $=0.0366$ ), and at the level of $1 \%$ in model 4 (i.e., $\beta=-0.1205$, $p$-value $=0.0078$ ). The negative sign with the coefficient depicts the negative effects of RNF on WPRD in the long run. However, the result portrays that 1-unit of increase in RNF brings about 0.3441 units of decrease in WPRD for the case of Northern region, while 0.1205 units of decrease in WPRD for the case of Southern region. Hence, (H6) is accepted for the case of the Central and Southern region only.

\section{Total sown area}

The coefficient of TSA is positive and significant at the level of $5 \%$ in model 1 (i.e., $\beta=1.0809, p$-value $=0.045$ ) and model 4 (i.e., $\beta=1.9664$, $p$-value $=3.0664$ ) and at the level of $1 \%$ in model 2 (i.e., $\beta=2.6908$, $p$-value $=0.0034$ ) and model 3 (i.e., $\beta=2.5443$, $p$-value $=0.0068$ ). The result indicates the positive impact of TSA on WPRD in the long run. The result further indicated that a 1-unit of increase in TSA would increase 1.0809, 2.6908, 2.5443, and 1.9664 units of WPRD for the case of Central, Northern, Western, and Southern region respectively. Results supported (H7) for the selected four regions. Figure 4A-4D indicates the visual expressions of the all relationships.

\section{Total un-irrigated area}

The coefficient of TUIRA is also significant and positive in model 1 - model 4, which reveals the positive long-run impact of TUIRA on WPRD. The result indicates that a 1-unit increase in TUIRA brings 1.9333 units of increase in WPRD for the case of the central region, while 2.1873, 2.8813, and 2.1784 units of increase for the case of Northern, western, and Southern region, respectively. (H8) is also supported for the selected four regions.

\section{Total irrigated area}

The coefficient of TIRA is $2.645,2.6149,2.3174$, and 1.8034 in models $1,2,3$, and 4 respectively. The positive sign and the significant p-values (i.e., 0.0041 , $0.02463,0.0071$, and 0.0274 , respectively) depict a positive association between TIRA and WPRD in the long run. The result portrays that 1 -unit of increase in TIRA will increase 2.645 units of WPRD in the central region, 2.6149 units of WPRD in the northern region, while 2.3174 and 1.8034 units of WPRD in the Western and Southern region. The results supported (H9) for the case of selected four regions 
Table 7

Long run Results (ARDL)

\begin{tabular}{|c|c|c|c|c|}
\hline DV: WPRD & Models/Regions & & & \\
\hline Independent Variables & Model 1: Central & Model 2: Northem & Model 3: Western & Model 4: Southern \\
\hline \multirow[t]{2}{*}{ WCLA } & $0.4545^{\star}$ & $1.3875^{\star \star \star}$ & 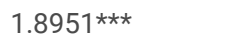 & $1.0652^{\star \star \star}$ \\
\hline & $(0.0734)$ & $(0.0005)$ & $(0.0000)$ & $(0.0094)$ \\
\hline \multirow[t]{2}{*}{ TMEAN } & $-1.0653^{\star \star}$ & $-1.1583^{\star \star \star}$ & $-0.9964^{\star \star}$ & -1.3776 ** \\
\hline & $(0.0255)$ & $(0.0017)$ & $(0.0341)$ & $(0.0272)$ \\
\hline \multirow[t]{2}{*}{ TMAX } & $-3.3287 \star \star \star$ & 1.0711 & 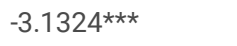 & $-1.4222^{\star \star}$ \\
\hline & $(0.0006)$ & $(0.1244)$ & $(0.0379)$ & $(0.0281)$ \\
\hline \multirow[t]{2}{*}{ TMIN } & $-1.8413^{* \star}$ & $-2.0740 \star \star \star *$ & -0.4301 & $-1.0949 *$ \\
\hline & $(0.0359)$ & $(0.0045)$ & $(0.2228)$ & $(0.078)$ \\
\hline \multirow[t]{2}{*}{ WND } & $-0.2176^{\star \star}$ & 1.4849 & $-0.2109 *$ & $-0.1085^{\star \star}$ \\
\hline & $(0.0269)$ & $(0.7354)$ & $(0.093)$ & $(0.0268)$ \\
\hline \multirow[t]{2}{*}{ RNF } & 0.3000 & $-0.3441 * \star$ & 0.1363 & $-0.1205^{\star \star \star}$ \\
\hline & $(0.1254)$ & $(0.0366)$ & $(0.4548)$ & $(0.0078)$ \\
\hline \multirow[t]{2}{*}{ TSA } & $1.0809 * \star$ & $2.6908^{* \star \star}$ & $2.5443^{\star \star \star}$ & 1.9664 ** \\
\hline & $(0.045)$ & $(0.0034)$ & $(0.0068)$ & $(0.0392)$ \\
\hline \multirow[t]{2}{*}{ TUIRA } & $1.9333^{* \star *}$ & $2.1873^{\star}$ & $2.8813^{\star \star *}$ & $2.1784^{\star \star}$ \\
\hline & $(0.0095)$ & $(0.0934)$ & $(0.008)$ & $(0.0353)$ \\
\hline \multirow[t]{2}{*}{ TIRA } & $2.6645^{\star \star \star}$ & $2.6149 * \star$ & $2.3174^{\star \star \star}$ & 1.8034 ** \\
\hline & $(0.0041)$ & $(0.0243)$ & $(0.0071)$ & $(0.0276)$ \\
\hline R-Square & 0.8645 & 0.7983 & 0.7672 & 0.8745 \\
\hline Adjusted R-Square & 0.7784 & 0.6973 & 0.6757 & 0.7653 \\
\hline
\end{tabular}

\section{Adjusted R-square}

The value of the adjusted R-square of model 1 shows that $77.84 \%$ variations in WPRD are collectively explained by all the independent variables for the case of the Central region. In contrast, in model 2, the value of adjusted R-square showed that in the Northern region, all the independent variables are explained $69.73 \%$ variations in WPRD. On the contrary, these variations ate $67.57 \%$ for the case of the Western region (see model 3 ) and $76.53 \%$ for the case of the Southern region (see model 4). Figure 4 indicates the Visual representation of the relationship between dependent and independent variable in various regions of Punjab.

\subsection{Model Stability}

The present study tests the stability of models for selected four regions by applying the CUSUM and CUSUM of the square test, suggested by Brown et al. (1975). Results are presented in Fig. 5A-5D (for the CUSUM test) and Fig. 6A-6D (for the CUSUM square test). Result reveals that the models for the present study are stable correctly specified as all the plots remain in the critical bounds.

\section{Conclusion And Policy Implications}

This study utilized ARDL bound test to investigate the impact of climate changes on food production in a different region of Punjab, Pakistan. This study found that Wheat cultivated area, total irrigated area, Total un-irrigated area, the total area sown, wind speed and rainfall has positive and significant impact on food production. Results exhibits that as Wheat cultivated area and total area sown, total irrigated and un-irrigated area, and rainfall increase in central Punjab the food production increases and Pakistan become self-sufficient in food production. However, average temperature, maximum, and minimum temperature hurts wheat production in central Punjab. Similarly, in northern Punjab, Wheat cultivated area, total irrigated area, Total un-irrigated area total area sown, wind speed, and the maximum temperature have a positive and significant impact on food production. In contrast, minimum temperature, average temperature, and rainfall have a negative association with food production.

Moreover, in western Punjab, Wheat cultivated area, total irrigated area, total un-irrigated area, the total area sown, rainfall and the average temperature have a positive association with wheat production. In contrast, max temperature, minimum temperature, and wind speed hurt food production. Similarly, in southern 
Punjab, wheat cultivated area, total irrigated area, Total un-irrigated area, and total area sown has a positive impact on food production while average, minimum, and maximum temperature, and wind speed harm food production.

The findings of this study suggest that the total wheat cultivated area should be increased to increase food production, so government should give incentives to the farmer for more production of Wheat. Similarly, the government should improve and upgrade the canal irrigation system to increase irrigated area and total sown area. Moreover, the findings of the study suggest that temperature negatively affect wheat production. However, to increase food production government should motivate individuals and organization large scale plantation to control the rapid increase in temperature.

\section{Declarations}

\section{Ethical Approval}

All authors read and approved the final manuscript.

\section{Consent to Participate}

Not applicable for that specific section

\section{Consent to Publish}

Not applicable for that specific section

\section{Author contributions}

Sohail Abbas, Shazia Kousar and Mahr Sahibzad Khan contributed to the study conception, design, data collection and analysis. Material preparation was also performed by Sohail Abbas. The first draft of the manuscript was written by Shiazia Kousar and Sohail Abbas.

\section{Funding information}

Not applicable for that specific section

\section{Conflict of interest}

All authors have no conflict of interest.

\section{Availability of data and materials}

Not applicable for that specific section

\section{Statement on Preprint Server}

We have not submitted my manuscript to a preprint server before submitting it to Environmental Science and Pollution Research

\section{Acknowledgements}

The data used in this study were collected from the Pakistan Meteorological Department (PMD) Lahore, Punjab Crop Reporting Service, Islamabad. We gratefully wish to thank these organizations for their valuable contributions during the collection of data.

\section{References}

1. Abbas, M., Sheikh, A. D., Shahbaz, M., \& Afzaal, A. (2007). Food security through wheat productivity in Pakistan. Sarhad Journal of Agriculture, 23(4), 1239.

2. Abbas, S., \& Dastgeer, G. (2021b). Analysing the impacts of climate variability on the yield of Kharif rice over Punjab, Pakistan. In Natural Resources Forum. Oxford, UK: Blackwell Publishing Ltd.

3. Abbas, S., Kousar, S., Shirazi, S. A., Yaseen, M., \& Latif, Y. (2021a). Illuminating Empirical Evidence of Climate Change: Impacts on Rice Production in the Punjab Regions, Pakistan. Agricultural Research, 1-16.

4. Abbas, S., \& Kousar, S. (2021d). Spatial analysis of drought severity and magnitude using the standardized precipitation index and streamflow drought index over the Upper Indus Basin, Pakistan. Environment, Development and Sustainability, 1-27.

5. Abbas, S., \& Mayo, Z. A. (2021c). Impact of temperature and rainfall on rice production in Punjab, Pakistan. Environment, Development and Sustainability, 23(2), 1706-1728.

6. Abbas, S., Khan, K., \& Khan, A. A. (2016a). REED plus and their impact on Green Economy Development: implication for the sustainable forest development, Swat Valley, HKH region Khyber PakhtunKhwa, Pakistan. Science International (Lahore), 28(5), 4657-4664.

7. Abbas, S., Khan, K., \& Ali, Z. (2016b). Green economic growth: an opportunity for sustainability and poverty alleviation, HKH region, Pakistan. Sci. Int, Lahore, 28(4), 3715-3721. 
8. Abdullah, M. H., Saboor, A., Baig, I. A., \& Arshad, M. (2016). Climate change, risk and food security: an analysis of wheat crop in Pakistan. In Climate Change Challenge (3C) and Social-Economic-Ecological Interface-Building (pp. 41-63). Springer, Cham.

9. Abid, S., Masood, M. A., Anwar, M. Z., Zahid, S., \& Raza, I. (2018). Trends and variability of wheat crop in Pakistan. Development, 8(2), 153-159.

10. Adnan, S., Ullah, K., Gao, S., Khosa, A. H., \& Wang, Z. (2017). Shifting of agroclimatic zones, their drought vulnerability, and precipitation and temperature trends in Pakistan. International Journal of Climatology, 37 (1), 529-543.

11. Ahmad, S., Abbas, G., Ahmed, M., Fatima, Z., Anjum, M. A., Rasul, G., ... \& Hoogenboom, G. (2019). Climate warming and management impact on the change of phenology of the rice-wheat cropping system in Punjab, Pakistan. Field crops research, 230, 46-61.

12. Ahmed, M., Akram, M. N., Asim, M., Aslam, M., Hassan, F. U., Higgins, S., ... \& Hoogenboom, G. (2016). Calibration and validation of APSIM-Wheat and CERES-Wheat for spring wheat under rainfed conditions: Models evaluation and application. Computers and Electronics in Agriculture, $123,384-401$.

13. Ali, S., Liu, Y., Ishaq, M., Shah, T., llyas, A., \& Din, I. U. (2017). Climate change and its impact on the yield of major food crops: Evidence from Pakistan. Foods, 6 (6), 1-19.

14. Arshad, M., Amjath-Babu, T. S., Krupnik, T. J., Aravindakshan, S., Abbas, A., Kachele, H., \& Miiller, K. (2017). Climate variability and yield risk in South Asia's rice-wheat systems: emerging evidence from Pakistan. Paddy and Water Environment, 15(2), 249-261.

15. Ashfaq, M., Zulfiqar, F., Sarwar, I., Quddus, M. A., \& Baig, I. A. (2011). Impact of climate change on wheat productivity in mixed cropping system of Punjab. Soil \& Environment, 30(2).

16. Bokhari, S. A. A., Rasul, G., Ruane, A. C., Hoogenboom, G., \& Ahmad, A. (2017). The Past and Future Changes in Climate of the Rice-Wheat Cropping Zone in Punjab, Pakistan. Pakistan Journal of Meteorology, 13 (26), 9-23.

17. Brown, R. L., Durbin, J., \& Evans, J. M. (1975). Techniques for testing the constancy of regression relationships over time. Journal of the Royal Statistical Society: Series B (Methodological), 37(2), 149-163.

18. Cheema, A., Khalid, L., \& Patnam, M. (2008). The geography of poverty: Evidence from the Punjab. The Lahore Journal of Economics, 13 (1), $163-188$.

19. Dhamija, V., Shukla, R., Gornott, C., \& Joshi, P. K. (2020). Consistency in vulnerability assessments of wheat to climate change-A district-level analysis in India. Sustainability, 12(19), 8256

20. Gul, F., Ahmed, I., Ashfaq, M., Jan, D., Fahad, S., Li, X., ... \& Shah, S. A. (2020). Use of crop growth model to simulate the impact of climate change on yield of various wheat cultivars under different agro-environmental conditions in Khyber Pakhtunkhwa, Pakistan. Arabian Journal of Geosciences, $13(3), 1-14$.

21. Iqbal, N., Bakhsh, K. H. U. D. A., Maqbool, A. S. I. F., \& Ahmad, A. S. (2005). Use of the ARIMA model for forecasting wheat area and production in Pakistan. Journal of Agriculture and Social Sciences, 1(2), 120-122.

22. Janjua, P. Z., Samad, G., Khan, N. U., \& Nasir, M. (2010). Impact of climate change on wheat production: A case study of Pakistan [with comments]. The Pakistan Development Review, 799-822.

23. Kirby, M., Mainuddin, M., Khaliq, T., \& Cheema, M. J. M. (2017). Agricultural production, water use and food availability in Pakistan: Historical trends, and projections to 2050. Agricultural Water Management, 179, 34-46.

24. Nawaz, Z., Li, X., Chen, Y., Guo, Y., Wang, X., \& Nawaz, N. (2019). Temporal and Spatial Characteristics of Precipitation and Temperature in Punjab, Pakistan. Water, 11 (9), 1-23.

25. Ray, D. K., Gerber, J. S., MacDonald, G. K., \& West, P. C. (2015). Climate variation explains a third of global crop yield variability. Nature communications, 6 (1), 1-9.

26. Siddiqui, R., Samad, G., Nasir, M., \& Jalil, H. H. (2012). The impact of climate change on major agricultural crops: evidence from Punjab, Pakistan. The Pakistan Development Review, 261-274.

27. Sultana, H., Ali, N., Iqbal, M. M., \& Khan, A. M. (2009). Vulnerability and adaptability of wheat production in different climatic zones of Pakistan under climate change scenarios. Climatic Change, 94(1), 123-142.

28. Tariq, A., Tabasam, N., Bakhsh, K., Ashfaq, M., \& Hassan, S. (2014). Food security in the context of climate change in Pakistan. Pakistan Journal of Commerce and Social Sciences (PJCSS), 8(2), 540-550.

29. Ullah, S. (2017). Climate change impact on agriculture of Pakistan-A leading agent to food security. International Journal of Environmental Science and Natural Resources, 6 (3), 1-4.

30. Wang, B., Chen, C., Li Liu, D., Asseng, S., Yu, Q., \& Yang, X. (2015). Effects of climate trends and variability on wheat yield variability in eastern Australia. Climate Research, 64 (2), 173-186.

31. Wilder, A. R. (1999). The Pakistani voter, electoral politics and voting behavior in Punjab. Karachi: Oxford University Press. p.190

32. Yu, Q., Li, L., Luo, Q., Eamus, D., Xu, S., Chen, C., Wang, E., Liu, Ji., \& Nielsen, D. C. (2014). Year patterns of climate impact on wheat yields. International Journal of Climatology, 34 (2), 518-528.

33. Zulfiqar, F., \& Hussain, A. (2014). Forecasting wheat production gaps to assess the state of future food security in Pakistan. Journal of Food and Nutritional Disorders, 3(3), 2.

\section{Figures}



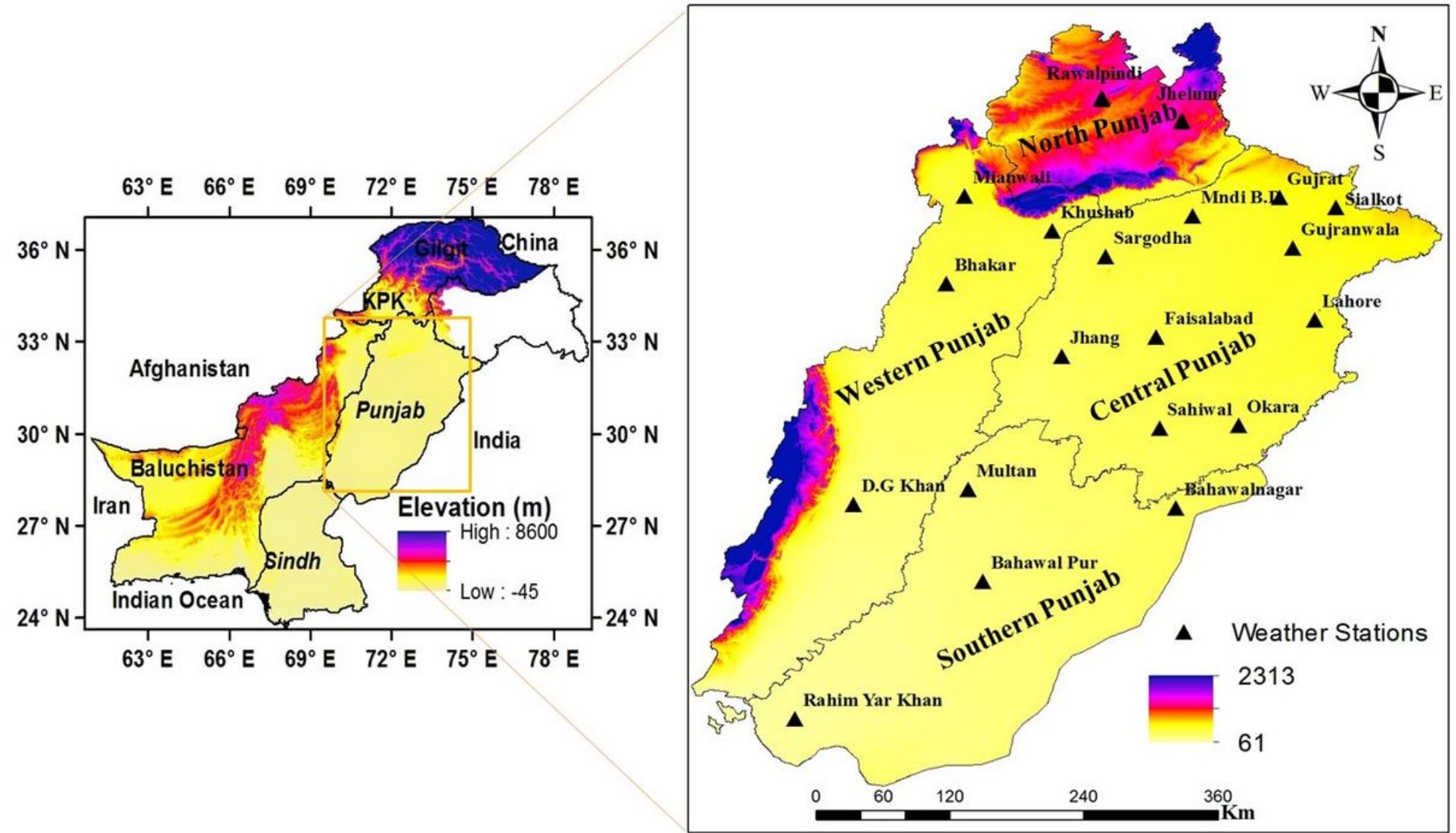

Figure 1

Location of the investigated area of the Punjab province, Pakistan. 


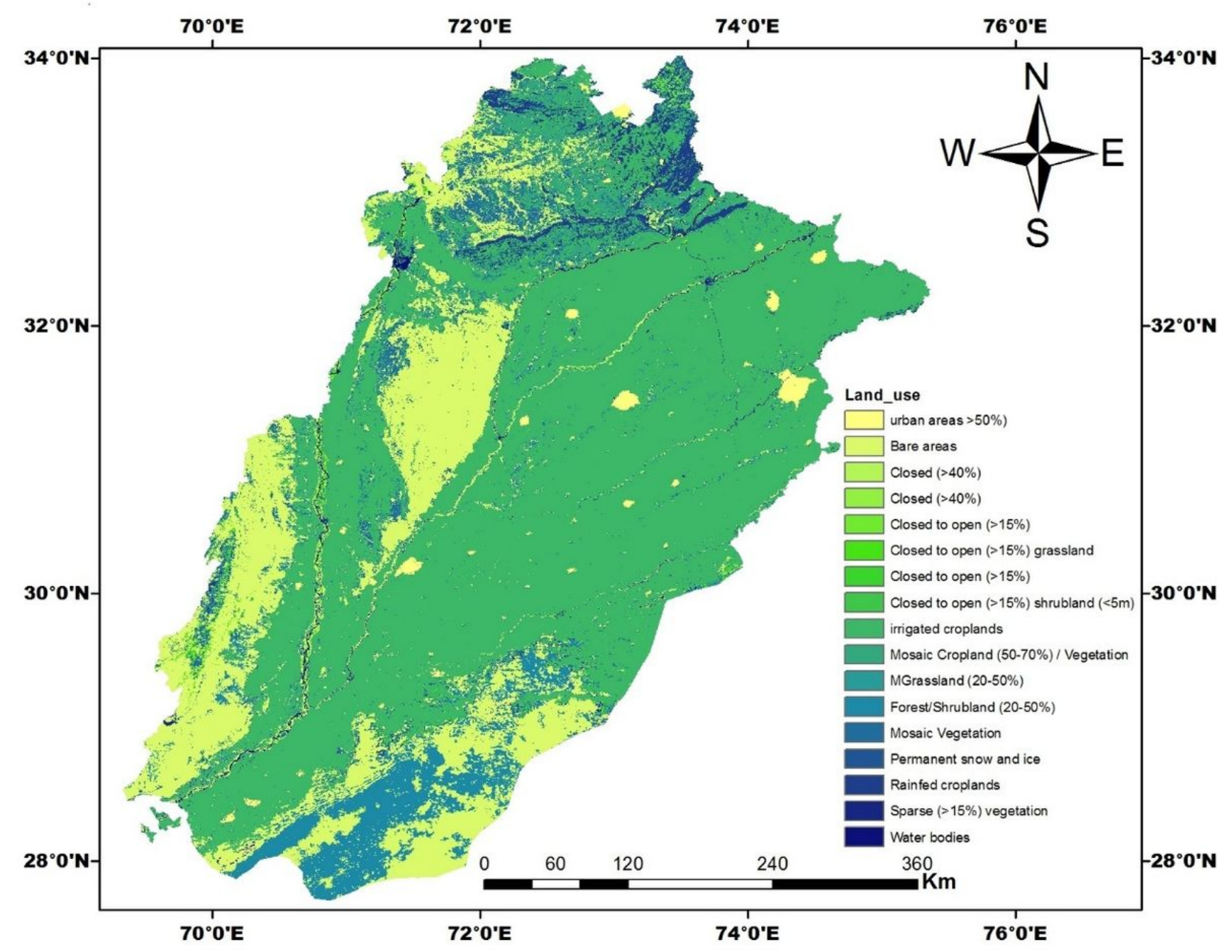

Figure 2

Land use patterns of the Punjab province, Pakistan. 


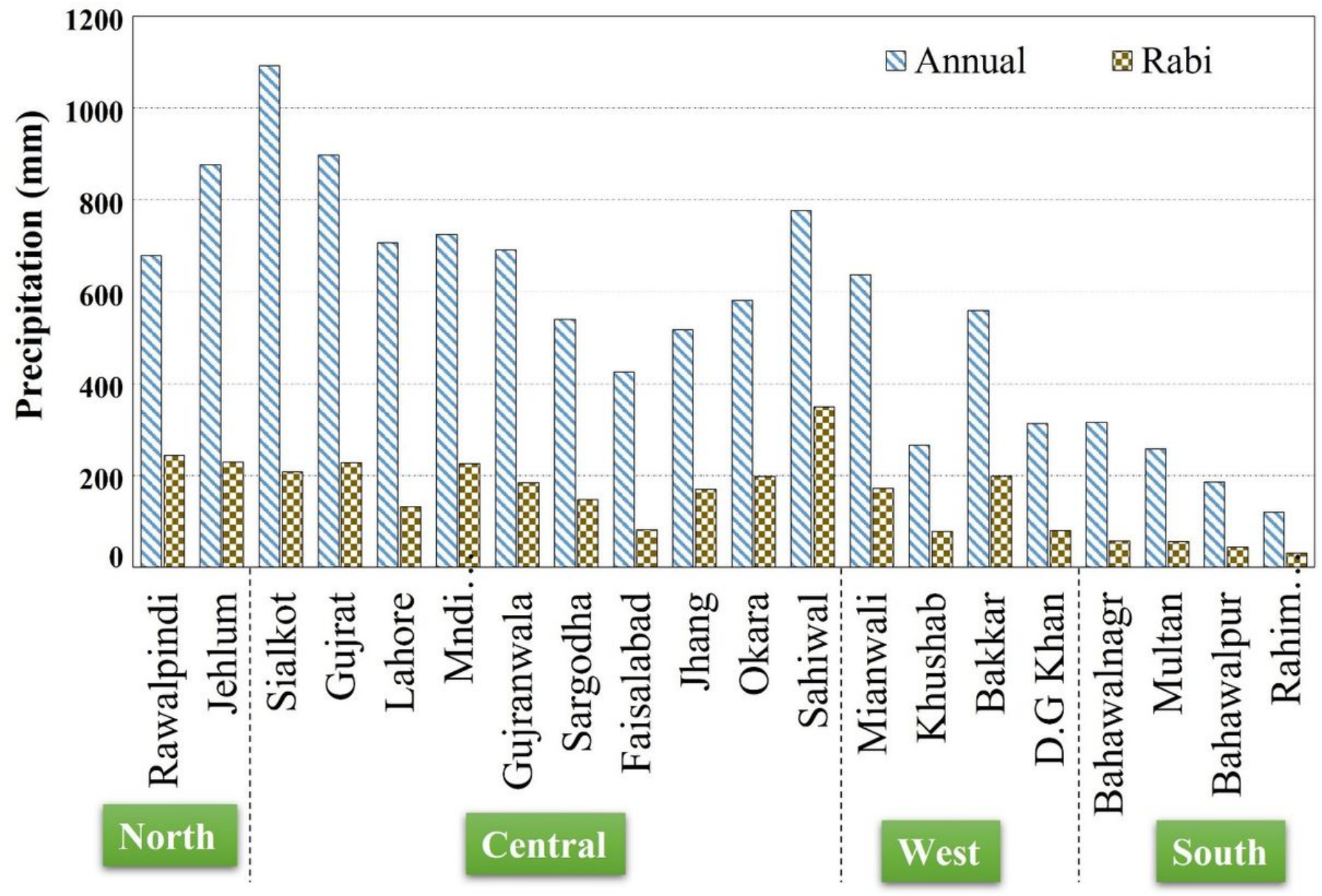

Figure 3

Annual and Rabi precipitation in the classified regions for 1979-2020. 
(A)

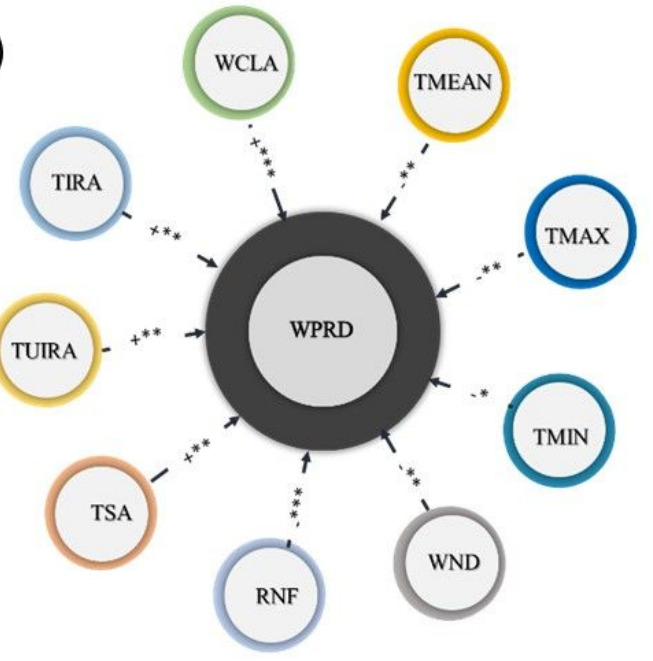

(C)

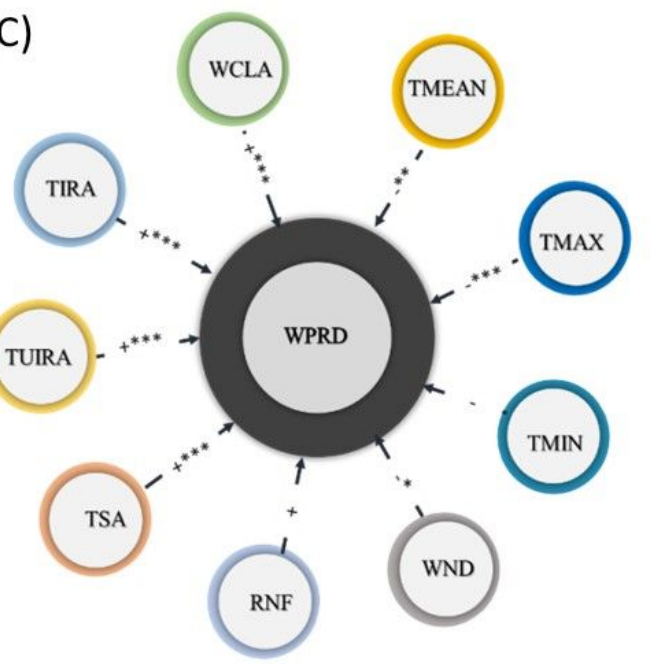

(B)
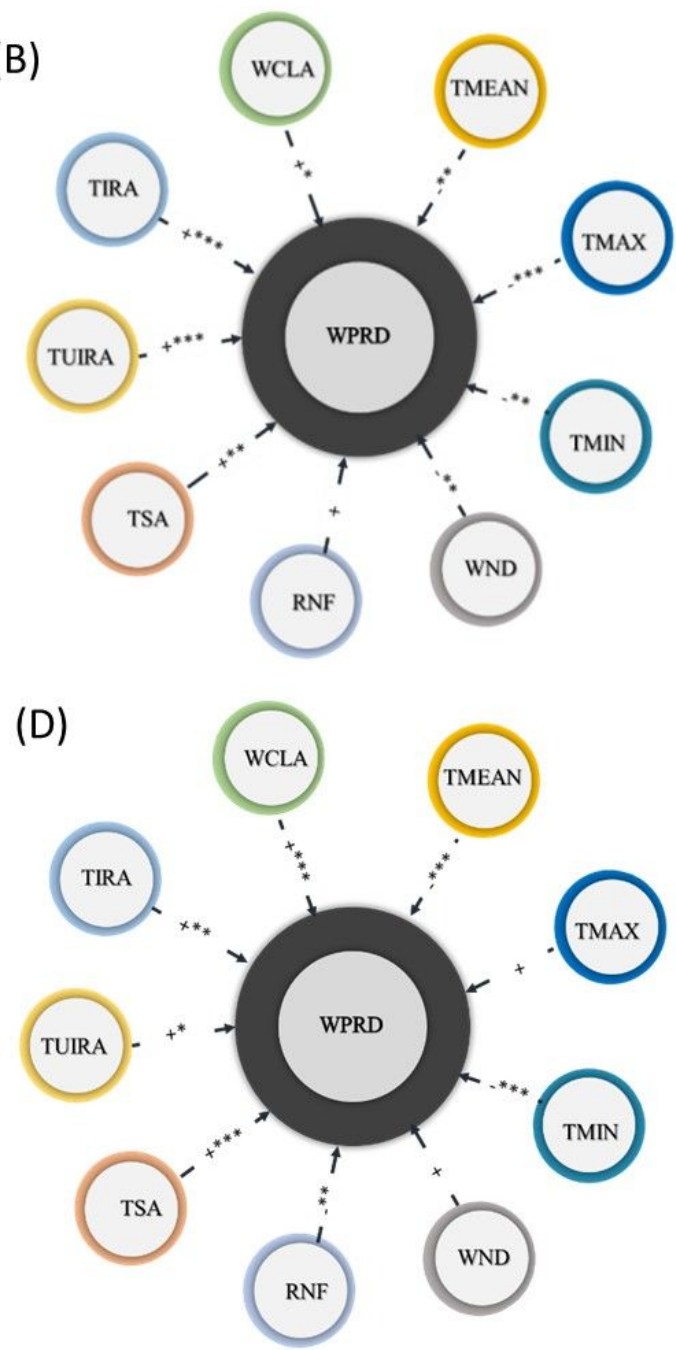

Figure 4

Visual representation of the relationship between dependent and independent variable (A) Central Punjab (b) Northern Punjab (C) Southern Punjab (D) Western Punjab Source: created by Author 

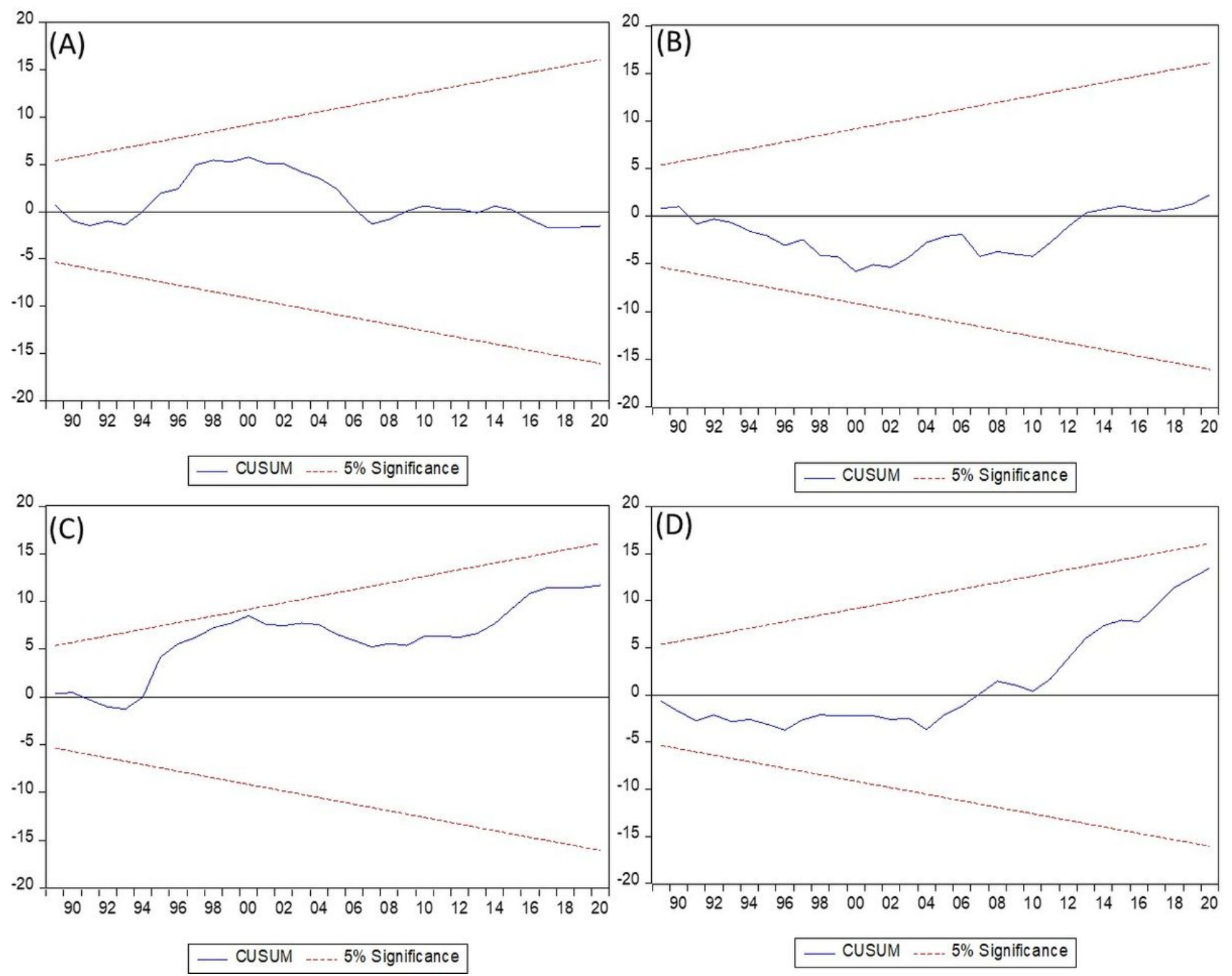

Figure 5

Stability of the model relationship for the CUSUM test (A) Central Punjab (b)Northern Punjab (C) Southern Punjab (D) Western Punjab Source: created by Author 

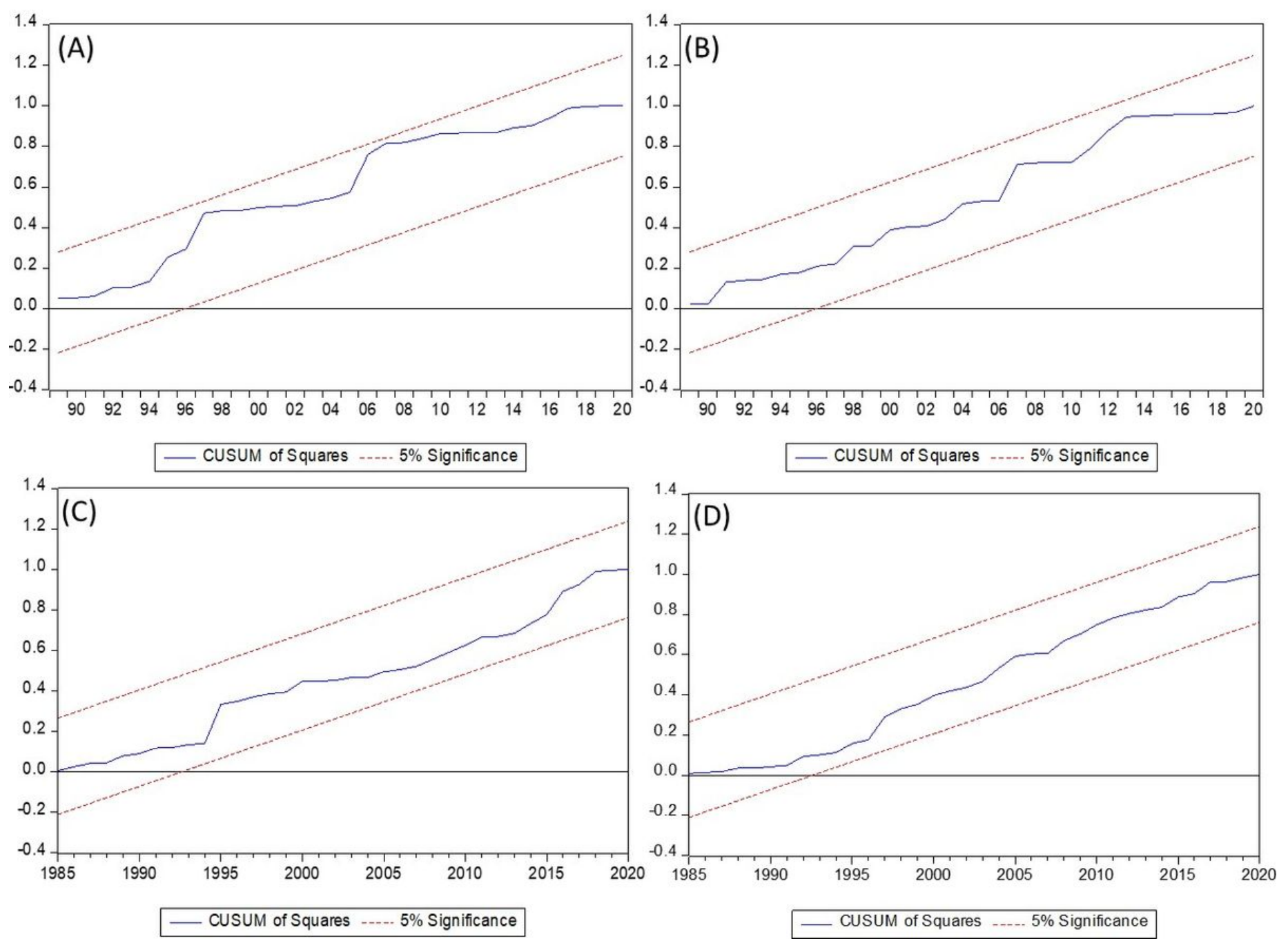

\section{Figure 6}

Stability of the model relationship for the CUSUM square test (A) Central Punjab (b)Northern Punjab (C) Southern Punjab (D) Western Punjab Source: created by Author 